\title{
Nonlocal Crowd Dynamics Models for Several Populations
}

\author{
Rinaldo M. Colombo ${ }^{1} \quad$ Magali Lécureux-Mercier ${ }^{2}$
}

November 11, 2018

\begin{abstract}
This paper develops the basic analytical theory related to some recently introduced crowd dynamics models. Where well posedness was known only locally in time, it is here extended to all of $\mathbb{R}^{+}$. The results on the stability with respect to the equations are improved. Moreover, here the case of several populations is considered, obtaining the well posedness of systems of multi-D non-local conservation laws. The basic analytical tools are provided by the classical Kružkov theory of scalar conservation laws in several space dimensions.
\end{abstract}

Keywords: Hyperbolic conservation laws, nonlocal flow, pedestrian traffic.

2010 MSC: 35L65

\section{Introduction}

From a macroscopic point of view, a crowd can be described through its density $\rho$, with $\rho \in \mathbf{L}^{1}\left(\mathbb{R}^{2} ; \mathbb{R}\right)$, and assuming that $\rho$ satisfies a continuity equation of the form

$$
\partial_{t} \rho+\operatorname{div}(\rho V)=0 .
$$

The vector $V$ is in general a function of the space coordinate $x \in \mathbb{R}^{2}$ and of the density $\rho$. The latter dependence may well be also of functional type since, in general, it is realistic to assume that $V$ depends on $\rho$ through some sort of weighted space average of $\rho$.

A first example of a model of this kind was presented in [6, Section 4]. There, it is assumed that pedestrians follow prescribed paths but adjust their speeds to the density they evaluate near to their positions. This amounts to postulate a speed law of the form:

$$
V=v(\rho * \eta) \vec{v}(x) .
$$

The integral curves of the vector field $\vec{v}$ are the trajectories followed by the pedestrians. For instance, $\vec{v}(x)$ is the unit tangent at $x$ to the geodesic curve joining $x$ to the destination of the pedestrian at $x$. The convolution $\rho * \eta$ stands for $\int_{\mathbb{R}^{2}} \eta(x-\xi) \rho(t, \xi) \mathrm{d} \xi$, for a suitable non-negative smooth kernel $\eta$. It represents the average density measured, or felt, by the pedestrian at time $t$ in position $x$.

Below, we extend the results in [6] proving the global in time existence of the solutions to (1.1) -(1.2). Moreover, we complete the stability estimates with an estimate on the dependence of the solutions from $v$ and $\vec{v}$, see Theorem 2.2. The resulting model (1.1)-(1.2) enjoys

\footnotetext{
${ }^{1}$ Department of Mathematics, Brescia University, Via Branze 38, 25133 Brescia, Italy

${ }^{2}$ Technion, Israel Institute of Technology, Amado Building, 32000 Haifa, Israel
} 
further remarkable analytical properties. Indeed, a standard conservation law generates a semigroup which is not differentiable with respect to the initial data see [3, Section 1] for an explicit example. On the contrary, under suitable conditions, the semigroup generated by (1.1) -(1.2) turns out to be differentiable with respect to the data. This allows to obtain, rigorously, necessary conditions for optimality in various control problems based on (1.1)(1.2), see Section 2 below and [6, Section 4].

Assuming that pedestrians adapt their path to the crowd density they meet lead to the model presented in [5]. There, the speed law

$$
V=v(\rho)(\vec{v}(x)+\mathcal{I}(\rho)) .
$$

is considered. Again, $\vec{v}$ is the unit vector field describing the preferred paths. But, contrary to (1.2), here pedestrians may deviate from it, due to the nonlocal term $\mathcal{I}$, which can be assumed, for instance, of the form

$$
\mathcal{I}(\rho)=-\varepsilon \frac{\nabla(\rho * \eta)}{\sqrt{1+\|\nabla(\rho * \eta)\|^{2}}} .
$$

Again, $(\rho * \eta)(t, x)$ is the average density felt by the pedestrian at $(t, x)$, so that (2.1) states that each individual is ready to leave the preferred path in order to avoid regions where the crowd density increases. The denominator in (1.4) is a normalization factor, so that the modulus of $V$ is essentially controlled by the function $v$ in (1.3), a smooth non increasing function that vanishes at the maximal density. We refer to [5] for further justifications of the choices leading to (1.1)-(1.3). Below, we show through numerical integrations that the formation of lanes, first noted in [5], is present also in the present multi-populations setting.

For both problems (1.1)-(1.2) and (1.1)-(1.3), we then consider the case of several, say $n$, populations. By this, we mean that different groups of pedestrians are considered, distinguished for instance by their destination. This amounts to consider systems of the form

$$
\partial_{t} \rho^{i}+\operatorname{div}\left(\rho^{i} V^{i}\right)=0, \quad i=1, \ldots, n
$$

where, in general, $V^{i}$ depends on the densities of all populations: $V^{i}=V^{i}\left(\rho_{1}, \ldots, \rho_{n}\right)$. To extend all the above well posedness and stability results, we rely here essentially on [7], with the improvements in [14]. We recall that systems of the form (1.5) are considered also in [8], where measure theoretic techniques are exploited. The interaction among different populations is considered also in [1] through a macroscopic model and in [9] by means of a multiscale model. For a general review about crowd dynamics models we refer to [2].

The next section deals with the $n$-populations version of (1.1)-(1.2). Then, Section 3 is devoted to the analogous extension of (1.1)-(1.3), presenting also a sample numerical integration. All proofs are collected in Section 4.

Throughout, we state and prove every result in $\mathbb{R}^{d}$, for a dimension $d \in \mathbb{N}, d>0$, since the $2 \mathrm{D}$ case contains the same difficulties as the general $d$-dimensional situation. By $\mathbb{R}^{+}$we denote the interval $[0,+\infty[$.

\section{A Differentiable Model}

The natural generalization of (1.2) to the case of $n$ populations is

$$
V^{i}=v^{i}\left(\rho^{1} * \eta^{1}+\ldots+\rho^{n} * \eta^{n}\right) \vec{v}^{i}(x),
$$


so that $\left(\rho^{i} * \eta^{i}\right)(x)$ is an average of the values attained by $\rho^{i}$ in $B(x, 1)$. The map $v^{i}$ is the usual speed law, typically required to be non increasing since at higher densities the mean traffic speed is lower. Below, only the regularity of $v$ is used. The vector $\vec{v}^{i}(x)$ is the direction of the pedestrian belonging to the $i$-th population and situated at $x \in \mathbb{R}^{2}$. The presence of boundaries, obstacles or other geometric constraints can be described through $\vec{v}^{i}$, see for instance [4].

This section is devoted to the well posedness of (1.5) -(2.1), extending the results in [6] not only for what concerns the number of populations, but also obtaining global in time existence and more complete stability estimates. Before stating the main result, we rigorously specify what we mean by solution.

Definition 2.1. Let $T>0$. Fix $\rho_{o} \in \mathbf{L}^{\infty}\left(\mathbb{R}^{d} ; \mathbb{R}^{n}\right)$. A weak entropy solution to (1.5)-(2.1) on $[0, T]$ is a bounded measurable map $\rho \in \mathbf{C}^{0}\left([0, T] ; \mathbf{L}_{l o c}^{1}\left(\mathbb{R}^{d} ; \mathbb{R}^{n}\right)\right)$ whose $i$-th component $\rho^{i}$, for all $i=1, \ldots, n$, is a Kružkov solution to the problem

$$
\left\{\begin{array}{l}
\partial_{t} \rho^{i}+\operatorname{div}\left(\rho^{i} V^{i}(t, x)\right)=0 \quad \text { where } \quad V^{i}(t, x)=v^{i}\left(\rho^{1}(t) * \eta^{1}+\ldots+\rho^{n}(t) * \eta^{n}\right) \vec{v}^{i}(x) . \\
\rho^{i}(0, x)=\rho_{o}^{i}(x)
\end{array} \quad\right. \text {. }
$$

For the definition of Kružkov solution, see [13] or [11, Paragraph 6.2]. Above, the convolution products in the arguments of $v^{i}$ are intended in the sense

$$
\left(\rho^{i}(t) * \eta^{i}\right)(x)=\int_{\mathbb{R}^{d}} \rho^{i}(t, \xi) \eta^{i}(x-\xi) \mathrm{d} \xi \quad i=1, \ldots, n .
$$

The next Theorem summarizes various results in [6], particularized to (1.1)-(1.2) .

Theorem 2.2. Fix $d, n \in \mathbb{N}$ with $d, n>0$. Assume the following conditions:

(v) $v^{i} \in\left(\mathbf{C}^{2} \cap \mathbf{W}^{2, \infty}\right)(\mathbb{R} ; \mathbb{R})$ for $i=1, \ldots, n$.

$(\overrightarrow{\boldsymbol{v}}) \quad \vec{v}^{i} \in\left(\mathbf{C}^{2} \cap \mathbf{W}^{2,1}\right)\left(\mathbb{R}^{d} ; \mathbb{S}^{1}\right)$ for $i=1, \ldots, n$.

$(\boldsymbol{\eta}) \eta^{i} \in\left(\mathbf{C}^{2} \cap \mathbf{W}^{2, \infty}\right)\left(\mathbb{R}^{d} ;[0,1]\right)$ and $\left\|\eta^{i}\right\|_{\mathbf{L}^{1}}=1$ for $i=1, \ldots, n$.

Then, there exists a semigroup $S: \mathbb{R}^{+} \times\left(\mathbf{L}^{1} \cap \mathbf{L}^{\infty} \cap \mathbf{B V}\right)\left(\mathbb{R}^{d} ; \mathbb{R}^{n}\right) \rightarrow\left(\mathbf{L}^{1} \cap \mathbf{L}^{\infty} \cap \mathbf{B V}\right)\left(\mathbb{R}^{d} ; \mathbb{R}^{n}\right)$ such that:

1. For all $\rho_{o} \in\left(\mathbf{L}^{1} \cap \mathbf{L}^{\infty} \cap \mathbf{B V}\right)\left(\mathbb{R}^{d} ; \mathbb{R}^{n}\right)$, for all $t \geq 0$, the orbit $t \mapsto S_{t} \rho_{o}$ is the unique solution to (1.1) -(2.1) in the sense of Definition 2.1 with initial datum $\rho_{o}$. Furthermore, the map $t \mapsto S_{t} \rho_{o}$ is in $\mathbf{C}^{0}\left(\mathbb{R}^{+} ; \mathbf{L}^{1}\left(\mathbb{R}^{d} ; \mathbb{R}^{n}\right)\right)$.

2. For all $\rho_{o} \in\left(\mathbf{L}^{1} \cap \mathbf{L}^{\infty} \cap \mathbf{B V}\right)\left(\mathbb{R}^{d} ; \mathbb{R}^{n}\right)$, if $\rho_{o}^{i} \geq 0$ for $i=1 \ldots, n$, then $\left(S_{t} \rho_{o}\right)_{i} \geq 0$ for all $t>0$.

3. There exists a constant $\mathcal{L}$ such that for all $\rho_{o} \in\left(\mathbf{L}^{1} \cap \mathbf{L}^{\infty} \cap \mathbf{B V}\right)\left(\mathbb{R}^{d} ; \mathbb{R}^{n}\right)$, the corresponding solution satisfies for all $t \in \mathbb{R}^{+}$

$$
\operatorname{TV}(\rho(t)) \leq\left(\operatorname{TV}\left(\rho_{o}\right)+\mathcal{L} t\left\|\rho_{o}\right\|_{\mathbf{L}^{\infty}}\right) e^{\mathcal{L} t} \quad \text { and } \quad\|\rho(t)\|_{\mathbf{L}^{\infty}} \leq\left\|\rho_{o}\right\|_{\mathbf{L}^{\infty}} e^{\mathcal{L} t}
$$


4. Fix a positive $M$. Then there exists functions $L, \mathcal{A}_{\eta}, \mathcal{A}_{v}, \mathcal{A}_{\vec{v}} \in \mathbf{C}^{0}\left(\mathbb{R}^{+} ; \mathbb{R}^{+}\right)$such for all $\rho_{o, 1}, \rho_{o, 2}$ in $\mathbf{L}^{1}\left(\mathbb{R}^{d} ; \mathbb{R}^{n}\right)$ with $\max \left\{\left\|\rho_{o, i}\right\|_{\mathbf{L}^{1}},\left\|\rho_{o, i}\right\|_{\mathbf{L}^{\infty}}, \mathrm{TV}\left(\rho_{o, i}\right)\right\} \leq M$, for all $v_{1}, v_{2}$ satisfying $(\boldsymbol{v})$, for all $\vec{v}_{1}, \vec{v}_{2}$ satisfying $(\overrightarrow{\boldsymbol{v}})$ and for all $\eta_{1}, \eta_{2}$ satisfying $(\boldsymbol{\eta})$, the corresponding solutions $\rho_{1}, \rho_{2}$ satisfy, for all $t \in \mathbb{R}^{+}$,

$$
\begin{aligned}
\left\|\rho_{1}(t)-\rho_{2}(t)\right\|_{\mathbf{L}^{1}} \leq & (1+t L(t))\left\|\rho_{o, 1}-\rho_{o, 2}\right\|_{\mathbf{L}^{1}} \\
& +t \mathcal{A}_{\eta}(t)\left\|\eta_{1}-\eta_{2}\right\|_{\mathbf{W}^{1, \infty}}+t \mathcal{A}_{v}(t)\left\|v_{1}-v_{2}\right\|_{\mathbf{W}^{1, \infty}} \\
& +t \mathcal{A}_{\vec{v}}(t)\left(\left\|\vec{v}_{1}-\vec{v}_{2}\right\|_{\mathbf{L}^{\infty}}+\left\|\vec{v}_{1}-\vec{v}_{2}\right\|_{\mathbf{W}^{1,1}}\right) .
\end{aligned}
$$

5. More regular initial data imply more regular solutions, in the sense that

$$
\begin{array}{ll}
\rho_{o} \in\left(\mathbf{W}^{1,1} \cap \mathbf{L}^{\infty}\right)\left(\mathbb{R}^{d} ; \mathbb{R}^{n}\right) & \Longrightarrow \forall t \in \mathbb{R}^{+}, \quad \rho(t) \in \mathbf{W}^{1,1}\left(\mathbb{R}^{d} ; \mathbb{R}^{n}\right), \\
\rho_{o} \in \mathbf{W}^{1, \infty}\left(\mathbb{R}^{d} ; \mathbb{R}^{n}\right) & \Longrightarrow \forall t \in \mathbb{R}^{+}, \quad \rho(t) \in \mathbf{W}^{1, \infty}\left(\mathbb{R}^{d} ; \mathbb{R}^{n}\right) .
\end{array}
$$

Furthermore, there exists a positive constant $C$ such that

$$
\|\rho(t)\|_{\mathbf{W}^{1,1}} \leq(1+C t) e^{C t}\left\|\rho_{o}\right\|_{\mathbf{W}^{1,1}} \quad \text { and } \quad\|\rho(t)\|_{\mathbf{W}^{1, \infty}} \leq(1+C t) e^{C t}\left\|\rho_{o}\right\|_{\mathbf{W}^{1, \infty}} .
$$

6. If $v, \vec{v}$ and $\eta$ are of class $\mathbf{C}^{3}$, then

$$
\begin{array}{ll}
\rho_{o} \in\left(\mathbf{W}^{2,1} \cap \mathbf{L}^{\infty}\right)\left(\mathbb{R}^{d} ; \mathbb{R}^{n}\right) & \Longrightarrow \forall t \in \mathbb{R}^{+}, \quad \rho(t) \in \mathbf{W}^{2,1}\left(\mathbb{R}^{d} ; \mathbb{R}^{n}\right), \\
\rho_{o} \in \mathbf{W}^{2, \infty}\left(\mathbb{R}^{d} ; \mathbb{R}^{n}\right) & \Longrightarrow \forall t \in \mathbb{R}^{+}, \quad \rho(t) \in \mathbf{W}^{2, \infty}\left(\mathbb{R}^{d} ; \mathbb{R}^{n}\right),
\end{array}
$$

and for a suitable non-negative constant $C$, we have the estimates

$$
\|\rho(t)\|_{\mathbf{W}^{2,1}} \leq e^{C t}(1+C t)^{2}\left\|\rho_{o}\right\|_{\mathbf{W}^{2,1}}, \quad\|\rho(t)\|_{\mathbf{W}^{2, \infty}} \leq e^{C t}(1+C t)^{2}\left\|\rho_{o}\right\|_{\mathbf{W}^{2, \infty}} .
$$

7. If $v$ is of class $\mathbf{C}^{4}$, for any initial data $\rho_{o} \in\left(\mathbf{W}^{2, \infty} \cap \mathbf{W}^{2,1}\right)\left(\mathbb{R}^{d} ; \mathbb{R}^{n}\right), \sigma_{o} \in\left(\mathbf{W}^{1,1} \cap\right.$ $\left.\mathbf{L}^{\infty}\right)\left(\mathbb{R}^{d} ; \mathbb{R}^{n}\right)$ and for all time $t \in \mathbb{R}^{+}$the semigroup $S$ is strongly $\mathbf{L}^{1}$ Gâteaux differentiable in the direction $\sigma_{o}$. The derivative $\mathrm{D} S_{t}\left(\rho_{o}\right)\left(\sigma_{o}\right)$ of $S_{t}$ at $\rho_{o}$ in the direction $\sigma_{o}$ is

$$
\mathrm{D} S_{t}\left(\rho_{o}\right)\left(\sigma_{o}\right)=\Sigma_{t}^{\rho_{o}}\left(\sigma_{o}\right)
$$

where $\Sigma^{\rho_{o}}$ is the linear semigroup generated by the Kružkov solution to

$$
\left\{\begin{array}{lrl}
\partial_{t} \sigma^{i}+\operatorname{div}\left(\sigma^{i} V^{i}(\rho)+\rho^{i} \mathrm{D} V^{i}(\rho)(\sigma)\right)=0 & (t, x) \in I \times \mathbb{R}^{d} \\
\sigma^{i}(0, x)=\sigma_{o}^{i}(x) & x \in \mathbb{R}^{d}
\end{array}\right.
$$

where $\rho(t)=S_{t} \rho_{o}$ for all $t \in \mathbb{R}^{+}$and $V^{i}$ is defined in (2.1).

Note that the linear problem (2.2) is the equation that is obtained through a merely formal linearization of (1.5)-(2.1).

The above regularity results allow to state and prove the following necessary condition for optimality.

Proposition 2.3. Let $f \in \mathbf{C}^{1,1}\left(\mathbb{R}^{n} ; \mathbb{R}^{+}\right), \psi \in \mathbf{L}^{\infty}\left(\mathbb{R}^{+} \times \mathbb{R}^{d} ; \mathbb{R}^{+}\right)$and assume that the problem (1.1)-(2.1) satisfies the assumptions at 6. in Theorem 2.2. Denote by $S: \mathbb{R}^{+} \times\left(\mathbf{L}^{1} \cap\right.$ 
$\left.\mathbf{L}^{\infty}\right)\left(\mathbb{R}^{d} ; \mathbb{R}^{n}\right) \rightarrow\left(\mathbf{L}^{1} \cap \mathbf{L}^{\infty}\right)\left(\mathbb{R}^{d} ; \mathbb{R}^{n}\right)$ the semigroup generated by (1.5)-(2.1). Introduce the integral cost functional

$$
J\left(\rho_{o}\right)=\int_{\mathbb{R}^{d}} f\left(S_{t} \rho_{o}\right) \psi(t, x) \mathrm{d} x .
$$

Then, $J$ is strongly $\mathbf{L}^{\infty}$ Gâteaux differentiable in any direction $\sigma_{o} \in\left(\mathbf{W}^{1,1} \cap \mathbf{L}^{\infty}\right)\left(\mathbb{R}^{d} ; \mathbb{R}^{n}\right)$.

Moreover, let $\Sigma: \mathbb{R}^{+} \times\left(\mathbf{W}^{1,1} \cap \mathbf{L}^{\infty}\right)\left(\mathbb{R}^{d} ; \mathbb{R}^{n}\right) \rightarrow\left(\mathbf{W}^{1,1} \cap \mathbf{L}^{\infty}\right)\left(\mathbb{R}^{d} ; \mathbb{R}^{n}\right)$ be the linear semigroup generated by (2.2). Then,

$$
D J\left(\rho_{o}\right)\left(\sigma_{o}\right)=\int_{\mathbb{R}^{d}} f^{\prime}\left(S_{t} \rho_{o}\right) \Sigma_{t}^{\rho_{o}}\left(\sigma_{o}\right) \psi(t, x) \mathrm{d} x .
$$

If $\rho_{\star} \in\left(\mathbf{L}^{1} \cap \mathbf{L}^{\infty}\right)\left(\mathbb{R}^{d} ; \mathbb{R}^{n}\right)$ solves the problem

$$
\text { find } \rho_{\star} \in\left(\mathbf{L}^{1} \cap \mathbf{L}^{\infty}\right)\left(\mathbb{R}^{d} ; \mathbb{R}^{n}\right) \quad \text { such that } \quad J\left(\rho_{\star}\right)=\min _{\rho_{o} \in\left(\mathbf{L}^{1} \cap \mathbf{L}^{\infty}\right)\left(\mathbb{R}^{d} ; \mathbb{R}^{n}\right)} J(\rho)
$$

then, for all $\sigma_{o} \in\left(\mathbf{L}^{1} \cap \mathbf{L}^{\infty}\right)\left(\mathbb{R}^{d} ; \mathbb{R}^{n}\right)$,

$$
\int_{\mathbb{R}^{d}} f^{\prime}\left(S_{t} \rho_{\star}\right) \Sigma_{t}^{\rho_{\star}} \sigma_{o} \psi(t, x) \mathrm{d} x=0 .
$$

The proof directly follows from [6, Proposition 2.12 and Theorem 4.2] and is hence omitted.

Remark that (1.5)-(2.1) provides an environment where optimal control problems can be considered. On the other hand, no uniform upper bound in $\mathbf{L}^{\infty}$ is available.

\section{Pattern Formation}

In the case of $n$ populations trying to avoid each other, we are led to consider (1.5) with

$$
V^{i}=v^{i}\left(\rho^{i}\right)\left(\vec{v}^{i}(x)+\mathcal{I}^{i}\left(\rho^{1}, \ldots, \rho^{n}\right)\right) \quad \text { for } \quad i=1, \ldots, n,
$$

where $\mathcal{I}^{1}, \ldots, \mathcal{I}^{n}$ are suitable nonlocal functionals. According to (3.1), the velocity $V^{i}$ of the $i$-th population is the product of a scalar crowding factor $v^{i}\left(\rho^{i}\right)$ with a vector $\vec{v}^{i}(x)+$ $\mathcal{I}^{i}\left(\rho^{1}, \ldots, \rho^{n}\right)$, which is the sum of a preferred direction $\vec{v}^{i}(x)$ and a deviation $\mathcal{I}^{i}\left(\rho^{1}, \ldots, \rho^{n}\right)$. The scalar $v^{i}\left(\rho^{i}\right)$ approximately gives the modulus of the speed. A further standard condition on $v^{i}$ typically required in the engineering literature is that $v^{i}$ be weakly decreasing. However, this assumption is here not exploited. The unit vector field $\vec{v}^{i}$ can be, for instance, the vector tangent at $x$ to the geodesic that the individuals in the $i$-th population would follow to get to their destination, if unaffected by any other individual. The term $\mathcal{I}^{i}\left(\rho^{1}, \ldots, \rho^{n}\right)$ describes how the $i$-th population deviates from its preferred trajectory due to the interaction among individuals, both of the same and of different populations. In general, it is a nonlocal functional, since its value at any position $x$ depends on the population densities averaged over a neighborhood of $x$. The present setting generalizes the model in [5].

Below, we first address the main analytical properties of (1.5)-(3.1), such as the existence of solutions, their continuous dependence from the initial data and their stability with respect to $\vec{v}^{i}$ and $\mathcal{I}^{i}$. Then, a numerical integrations shows further qualitative properties of the solutions to (1.5)-(3.1).

Denote by $R>0$ a given maximal density. Throughout, we also denote the flux of the $i$-th population by $q^{i}\left(\rho^{i}\right)=\rho^{i} v^{i}\left(\rho^{i}\right)$, for all $\rho^{i} \in[0, R]$.

Our starting point is the rigorous definition of solution to (1.5)-(3.1), analogous to Definition 2.1. 
Definition 3.1. Fix the initial datum $\left(\rho_{o}^{1}, \ldots, \rho_{o}^{n}\right) \in\left(\mathbf{L}^{1} \cap \mathbf{L}^{\infty}\right)\left(\mathbb{R}^{d} ;[0, R]^{n}\right) . \quad A$ map $\rho \in$ $\mathbf{C}^{0}\left([0, T] ; \mathbf{L}^{1}\left(\mathbb{R}^{d} ;[0, R]^{n}\right)\right)$ is a weak entropy solution to (1.5)-(3.1) corresponding to the initial condition $\left(\rho_{o}^{1}, \ldots, \rho_{o}^{n}\right)$ if, for $i=1, \ldots, n, \rho^{i}$ is a Kružkov solution to the Cauchy problem for the scalar conservation law

$$
\left\{\begin{array}{l}
\partial_{t} \rho^{i}+\operatorname{div}\left(\rho^{i} v^{i}\left(\rho^{i}\right) V^{i}(t, x)\right)=0 \quad \text { where } \quad V^{i}(t, x)=\vec{v}^{i}(x)+\mathcal{I}^{i}\left(\rho_{1}(t), \ldots, \rho_{n}(t)\right)(x) . \\
\rho^{i}(0, x)=\rho_{o}^{i}(x)
\end{array} \quad\right. \text {. }
$$

For the definition of Kružkov solution we refer to [13, Definition 1].

Theorem 3.2. Assume the following conditions:

(v) For $i=1, \ldots, n, v^{i} \in \mathbf{C}^{2}\left([0, R] ; \mathbb{R}^{+}\right)$satisfies $v^{i}(R)=0$.

$(\overrightarrow{\boldsymbol{v}})$ For $i=1, \ldots, n, \vec{v}^{i} \in\left(\mathbf{C}^{2} \cap \mathbf{W}^{1, \infty}\right)\left(\mathbb{R}^{d} ; \mathbb{R}^{d}\right)$ and $\operatorname{div} \vec{v}^{i} \in \mathbf{W}^{1,1}\left(\mathbb{R}^{d} ; \mathbb{R}^{d \times d}\right)$.

(I) There exists a constant $C_{I}>0$ such that the functional $\mathcal{I}^{i}: \mathbf{L}^{1}\left(\mathbb{R}^{d} ;[0, R]^{n}\right) \rightarrow \mathbf{C}^{2}\left(\mathbb{R}^{d} ; \mathbb{R}^{d}\right)$ satisfies, for $i=1, \ldots, n$,

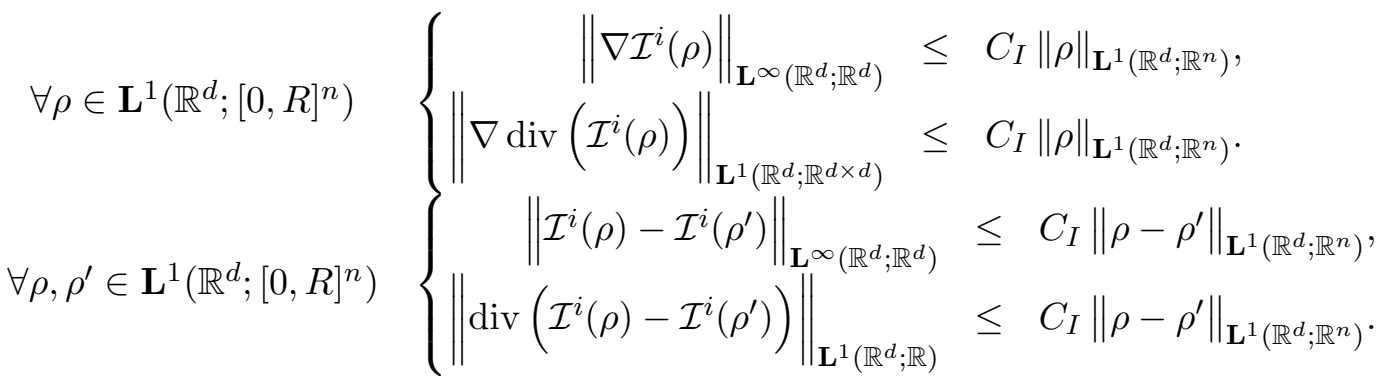

Then, there exists a semigroup $S: \mathbb{R}^{+} \times\left(\mathbf{L}^{1} \cap \mathbf{B V}\right)\left(\mathbb{R}^{d} ;[0, R]^{n}\right) \rightarrow\left(\mathbf{L}^{1} \cap \mathbf{B V}\right)\left(\mathbb{R}^{d} ;[0, R]^{n}\right)$ such that

1. For all $\rho_{o} \in\left(\mathbf{L}^{1} \cap \mathbf{B V}\right)\left(\mathbb{R}^{d} ;[0, R]^{n}\right)$, the orbit $t \mapsto S_{t} \rho_{o}$ is the unique solution to (1.1)(3.1) with initial datum $\rho_{o}$ in the sense of Definition 3.1.

2. For all $\rho_{o} \in\left(\mathbf{L}^{1} \cap \mathbf{B V}\right)\left(\mathbb{R}^{d} ;[0, R]^{n}\right)$, the map $t \mapsto S_{t} \rho_{o}$ is in $\mathbf{C}^{0}\left(\mathbb{R}^{+} ; \mathbf{L}^{1}\left(\mathbb{R}^{d} ;[0, R]^{n}\right)\right)$.

3. For all $\rho_{o} \in\left(\mathbf{L}^{1} \cap \mathbf{B V}\right)\left(\mathbb{R}^{d} ;[0, R]^{n}\right)$, the following estimate holds:

$$
\operatorname{TV}\left(S_{t} \rho_{o}\right) \leq \operatorname{TV}\left(\rho_{o}\right) e^{\kappa_{o} t}+d W_{d} e^{\kappa_{o} t}\|q\|_{\mathbf{L}^{\infty}([0, R])}\left(C_{I}+\|\operatorname{div} \vec{v}\|_{\mathbf{L}^{\infty}}\right) t,
$$

where $W_{d}$ is defined in (4.1).

4. Fix $M>0$. Let $v_{1}, v_{2}$ satisfy $(\boldsymbol{v}), \vec{v}_{1}, \vec{v}_{2}$ satisfy $(\boldsymbol{v})$ and $\mathcal{I}_{1}, \mathcal{I}_{2}$ satisfy $(\boldsymbol{I})$. Then, there exist $b, c \in \mathbf{C}^{0}\left(\mathbb{R}^{+} ; \mathbb{R}^{+}\right)$such that for all $\rho_{o, 1}, \rho_{o, 2} \in \mathbf{L}^{1}\left(\mathbb{R}^{d} ;[0, R]^{d}\right)$ with $\operatorname{TV}\left(\rho_{o, i}\right) \leq M$ and for all $t \in \mathbb{R}^{+}$

$$
\begin{aligned}
\left\|S_{t} \rho_{o, 1}-S_{t} \rho_{o, 2}\right\|_{\mathbf{L}^{1}} \leq & \left(1+t e^{t b(t)}\right)\left\|\rho_{o, 1}-\rho_{o, 2}\right\|_{\mathbf{L}^{1}} \\
& +t c(t)\left(\left\|q_{1}-q_{2}\right\|_{\mathbf{W}^{1, \infty}}+\left\|\vec{v}_{1}-\vec{v}_{2}\right\|_{\mathbf{L}^{\infty}}+\left\|\operatorname{div}\left(\vec{v}_{1}-\vec{v}_{2}\right)\right\|_{\mathbf{L}^{1}}\right) .
\end{aligned}
$$

and the functions $b, c$ depend on $d, C_{I}, R,\left\|q_{1}\right\|_{\mathbf{W}^{1, \infty}},\left\|\vec{v}_{1}\right\|_{\mathbf{W}^{1, \infty}},\left\|\operatorname{div} \vec{v}_{1}\right\|_{\mathbf{W}^{1,1}},\|\nabla \eta\|_{\mathbf{L}^{\infty}}$ and on $M$. 
Detailed expressions for the functions $b$ and $c$ are available, together with the proof, in Paragraph 4.2 .

This theorem also provides a kind of maximum property since each density remains bounded by $R$. However, it does not guarantee that the sum $\rho_{1}(t)+\ldots+\rho_{n}(t)$ remains bounded by $R$.

Preliminary to any use of (1.5) -(3.1) is the choice of a specific $\mathcal{I}$. First, we consider the following lemma that eases the construction of operators that satisfy (I).

Lemma 3.3. Let $\mathcal{N}: \mathbb{R}^{d} \rightarrow \mathbb{R}^{d}$ be defined by

$$
\mathcal{N}(u)=\frac{u}{\sqrt{1+\|u\|^{2}}} .
$$

If $\mathcal{I}$ satisfies (I), then so does $\mathcal{N} \circ \mathcal{I}$.

The proof consists in long but elementary computations and is hence omitted. By Lemma 3.3. it is immediate to check that (1.4) is satisfied by the operator $\mathcal{I}$ defined in (1.4) in the case of one population, as soon as $\eta \in \mathbf{C}_{c}^{2}\left(\mathbb{R}^{d} ; \mathbb{R}^{+}\right)$and $\|\eta\|_{\mathbf{L}^{1}}=1$, considered in $[6]$. When more populations are present, it is natural to consider different kinds of interactions. For instance, the population $\rho^{1}$ might deviate from its preferred path $\vec{v}^{1}$ due to the population $\rho^{2}$ pushing in the direction $\vec{v}^{2}$, thus leading to consider the operator

$$
\mathcal{I}^{12}(\rho)=\frac{\left(\rho^{2} v\left(\rho^{2}\right) \vec{v}^{2}\right) * \eta}{\sqrt{1+\left\|\left(\rho^{2} v\left(\rho^{2}\right) \vec{v}^{2}\right) * \eta\right\|^{2}}},
$$

Under assumption (v) on $v$ and with $\eta \in \mathbf{C}_{c}^{2}\left(\mathbb{R}^{d} ; \mathbb{R}^{+}\right)$such that $\|\eta\|_{\mathbf{L}^{1}}=1$, also $\mathcal{I}^{12}$ as defined in (3.3) satisfies (I).

When these or other operators are to be considered together, then the following lemma makes the verification of (I) immediate.

Lemma 3.4. Let $\hat{\mathcal{I}}$ and $\check{\mathcal{I}}$ satisfy (I). Fix any two functions $\alpha, \beta \in\left(\mathbf{C}^{2} \cap \mathbf{W}^{2, \infty}\right)\left(\mathbb{R}^{d} ; \mathbb{R}\right)$. Then, also $\alpha \hat{\mathcal{I}}+\beta \check{\mathcal{I}}$ satisfies (I).

The proof is elementary and hence omitted.

Differently from the model in Section 2, the semigroup generated by (1.5)-(3.1) is not proved to be differentiable with respect to the initial data. On other hand, it develops solutions with an apparently rich structure. Moreover, as stated in Theorem [3.2, an upper bound in $\mathbf{L}^{\infty}$ is available.

\subsection{Numerical Integration}

The study of the qualitative properties of the solutions to (1.5)-(3.1) is in general not amenable to purely analytical tools. Numerical integrations, besides being of interest from the point of view of the applications, show interesting pattern formations. In the case (1.1)-(1.3) of a single population, the formation of queues was already noted in 5 .

The algorithm used is the Lax-Friedrichs method with dimensional splitting. A uniform grid $\left(x_{i}, y_{j}\right)$ for $i=1, \ldots, n_{x}$ and $j=1, \ldots, n_{y}$ is introduced and the density $\rho$ is approximated 
through the values $\rho_{i j}$ on this grid. At every time step, the convolution in $\mathcal{I}(\rho)$ is computed through products of the type $A_{i h} \rho_{h k} B_{k j}$, where the matrices $A$ and $B$ depend only on $\eta$.

In both the integrations, we use the same geometry and the same numerical parameters. More precisely, the space available to the pedestrians is the rectangle $\mathbb{R} \times[-3,3]$, while the numerical domain is $[-8,8] \times[-4,4]$. Pedestrian may exit along the segments $\{-8\} \times[-3,3]$ and $\{8\} \times[-3,3]$. The mesh size is $\Delta x=\Delta y=0.025$.

The preferred path of each pedestrian is the sum $g+\delta$. The vector $g$ is tangent to the geodesic towards the pedestrian's target or 0 when the pedestrians would not move. The vector $\delta$ describes the discomfort of pedestrian when walking too near to a wall. It is perpendicular to the walls and pointing towards the interior of the room. Analytically, it also ensures the invariance of the room, see [5] for more details. Its maximum modulus is $\delta_{\max }$ along the walls at $\left|x_{2}\right|=\Delta$ and decreases linearly towards the room interior, vanishing at a distance $\delta_{r}$ from the walls. In the present integration we set

$$
\delta_{\max }=0.8, \quad \delta_{r}=0.75 .
$$

\subsection{Two Groups of People Crossing}

A classical situation considered in the engineering literature is that of two groups of people moving in opposite directions and crossing each other. Typically, lanes, also called paths or trails in the engineering literature, are formed, see for instance [12, 10] or [1] for a one dimensional description. They consist of people going in the same direction. The model (1.1)(2.1) captures this phenomenon. Indeed, we consider (1.5)-(2.1) in the following setting:

$$
\left\{\begin{array}{l}
\partial_{t} \rho^{1}+\operatorname{div}\left(\begin{array}{l}
\rho^{1} v\left(\rho^{1}\right)\left(\vec{v}^{1}(x)-\varepsilon_{11} \frac{\nabla\left(\rho^{1} * \eta\right)}{\sqrt{1+\left\|\nabla\left(\rho^{1} * \eta\right)\right\|^{2}}}-\varepsilon_{12} \frac{\nabla\left(\rho^{2} * \eta\right)}{\sqrt{1+\left\|\nabla\left(\rho^{2} * \eta\right)\right\|^{2}}}\right) \\
\partial_{t} \rho^{2}+\operatorname{div}\left(\rho^{2} v\left(\rho^{2}\right)\left(\vec{v}^{2}(x)-\varepsilon_{21} \frac{\nabla\left(\rho^{1} * \eta\right)}{\sqrt{1+\left\|\nabla\left(\rho^{1} * \eta\right)\right\|^{2}}}-\varepsilon_{22} \frac{\nabla\left(\rho^{2} * \eta\right)}{\sqrt{1+\left\|\nabla\left(\rho^{2} * \eta\right)\right\|^{2}}}\right)\right.
\end{array}\right)=0
\end{array}\right.
$$

where the $\rho^{1}$ population moves to the right and the $\rho^{2}$ population move to the left. Above, we assume that each pedestrians wants to avoid entering regions with increasing densities and that the repulsion towards pedestrians of the other population is bigger than that due to the proper population. For the sake of simplicity, we use the same kernel $\eta$ and the same speed $v$ in both equations. We consider the specific situation defined by

$$
\begin{aligned}
& \vec{v}^{1}=\left[\begin{array}{l}
1 \\
0
\end{array}\right]+\delta, \quad \eta\left(x_{1}, x_{2}\right)=\left[1-\left(2 x_{1}\right)^{2}\right]^{3}\left[1-\left(2 x_{2}\right)^{2}\right]^{3} \chi_{[-0.5,0.5]^{2}}\left(x_{1}, x_{2}\right), \\
& \vec{v}^{2}=\left[\begin{array}{c}
-1 \\
0
\end{array}\right]+\delta, \quad v(\rho)=4(1-\rho), \quad \begin{array}{l}
\varepsilon_{11}=0.3, \quad \varepsilon_{12}=0.7 \\
\varepsilon_{21}=0.7, \quad \varepsilon_{22}=0.3 .
\end{array}
\end{aligned}
$$

As initial datum we choose

$$
\begin{aligned}
& \rho_{o}^{1}\left(x_{1}, x_{2}\right)=0.9 \cdot \chi_{[-6.4,-3.2] \times[-2.4,2.4]}\left(x_{1}, x_{2}\right), \\
& \rho_{o}^{2}\left(x_{1}, x_{2}\right)=0.7 \cdot \chi_{[3.2,6.4] \times[-2.4,2.4]}\left(x_{1}, x_{2}\right) .
\end{aligned}
$$


The resulting numerical integration in Figure 1 shows several lanes forming. First, when most of the populations are still separated, the lanes in the two populations are rather symmetric, in spite of the different initial densities Then, when the interaction between $\rho^{1}$ and $\rho^{2}$ gets
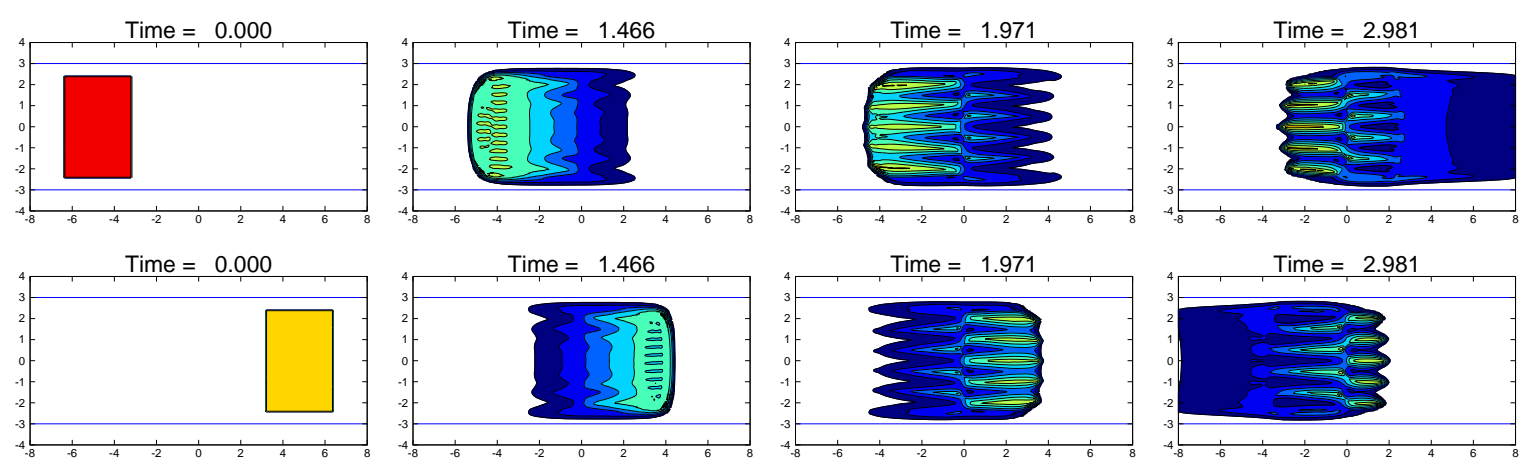

Figure 1: Numerical integration of (3.5) with data (3.7) and parameters as in (3.4)-(3.6). Above, the population $\rho^{1}$ moving to the right and, below, $\rho^{2}$ moving to the left. Note the lanes that are formed. First, due to the self-interaction within each populations and then due to the crossing between the two populations. The latter lanes of different populations do not superimpose.

relevant, lanes sharply bend their trajectory so that they are not superimposed. In this way, pedestrians lower how much they block each other. After the interaction, the density is so low that no more patterns arise.

\subsection{Part of an Audience Leaving a Room}

A sample situations developing various interesting features is the following. Two populations are initially uniformly distributed in the same region. At time $t=0$, the first populations starts moving towards an exit, on the right in Figure 21), while the second moves only to let the first one pass. We describe this situation through (1.5)-(3.1). The preferred path of $\rho^{1}$ is tangent to $\vec{v}^{1}=g+\delta$, where $g$ is the geodesic vector field directed toward the right exit and $\delta$ is the discomfort, as above. The $\rho^{1}$ population then deviates from this trajectory only to avoid the other population. On the contrary, the preferred path of $\rho^{2}$ is tangent to $\vec{v}^{2}=\delta$, since this population moves only to avoid the walls and the $\rho^{1}$ population. All this leads to the system

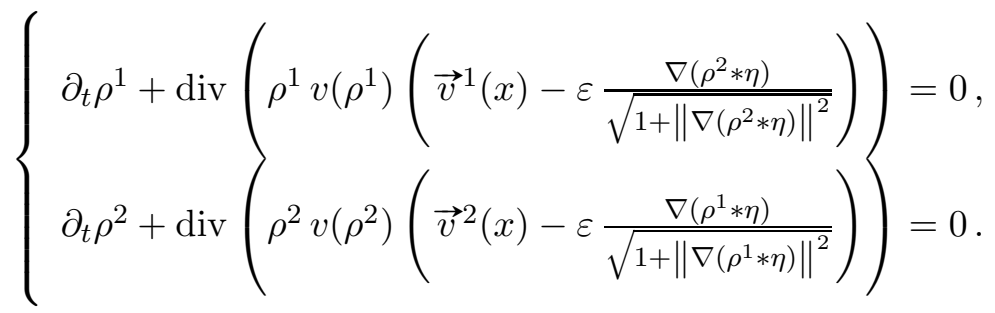

We consider the specific situation defined by

$$
\begin{aligned}
& \vec{v}^{1}=\left[\begin{array}{l}
1 \\
0
\end{array}\right]+\delta, \quad \eta\left(x_{1}, x_{2}\right)=\left[1-\left(2 x_{1}\right)^{2}\right]^{3}\left[1-\left(2 x_{2}\right)^{2}\right]^{3} \chi_{[-0.5,0.5]^{2}}\left(x_{1}, x_{2}\right), \\
& \vec{v}^{2}=\delta, \quad v(\rho)=4(1-\rho), \quad \varepsilon=0.3 .
\end{aligned}
$$


with initial data

$$
\rho_{o}^{i}=0.5 \chi_{[-6.4,-3.2] \times[-2.4,2.4]}, \quad i=1,2,
$$

The resulting numerical integration is in Figure 2. Note that the initial distributions of
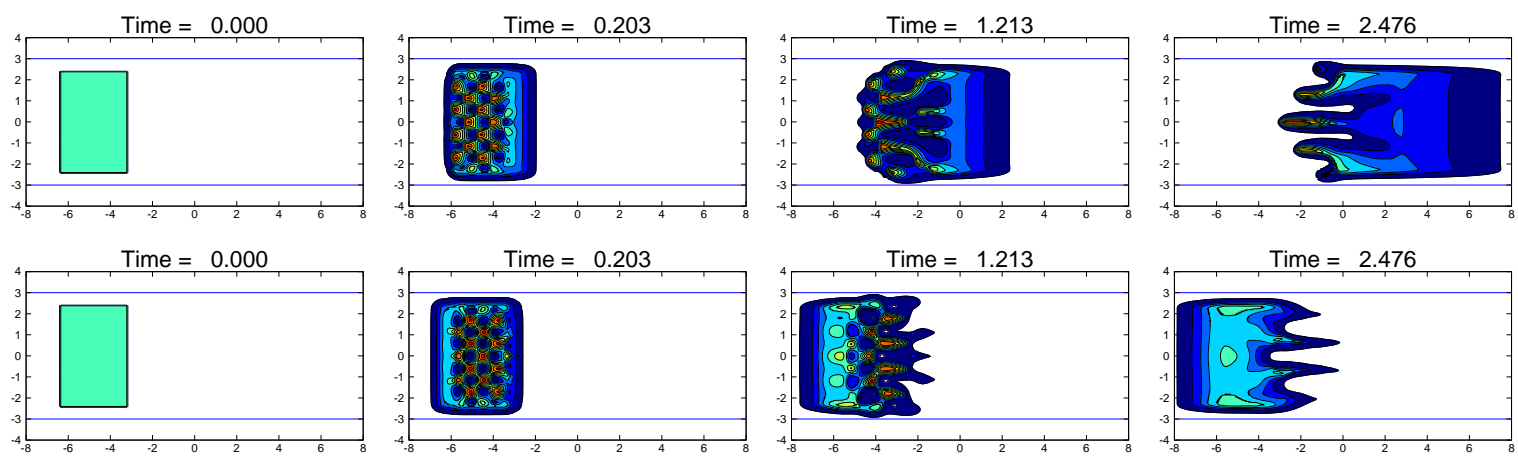

Figure 2: Numerical integration of (3.8) with data and parameters as in (3.4)-(3.9). Above, the population $\rho^{1}$ and, below, $\rho^{2}$. Note first the formation of small clusters separating the two populations, then lanes and, finally, a sort of fingering.

both populations are uniform. Very quickly, patterns start to form. First, clustering: the different populations separate forming separated peaks of high density. Then, population $\rho^{1}$ starts moving significantly to the right along lanes that follow paths in regions of low $\rho^{2}$ density. Finally, the two populations are almost separated, with a sort of fingering remaining in the region when they are superimposed. Concerning the initial clustering, we underline the analogy with [9], where a population is described through a measure possibly containing also an atomic part. Concerning the latter fingering, we borrowed here this term from the entirely different case of, for instance, thin films, see [15]).

\section{Technical Details}

Below, for any $\rho \in \mathbf{L}^{1}\left(\mathbb{R}^{d} ; \mathbb{R}^{n}\right),\|\rho\|_{\mathbf{L}^{1}\left(\mathbb{R}^{d} ; \mathbb{R}^{n}\right)}$ stands for $\sum_{i=1}^{n}\left\|\rho^{i}\right\|_{\mathbf{L}^{1}\left(\mathbb{R}^{d} ; \mathbb{R}\right)}$. Similarly, TV $(\rho)=$ $\sum_{i=1}^{n} \mathrm{TV}\left(\rho^{i}\right)$. More generally, if not otherwise stated, the norm of a vector $v$ in $\mathbb{R}^{n}$ is the 1-norm, i.e. $\|v\|=\sum_{i=1}^{n}\left|v^{i}\right|$. The following constant enters several estimates below:

$$
W_{d}=\int_{0}^{\pi / 2}(\cos \theta)^{d} \mathrm{~d} \theta
$$

The analytical tools below are based on the classical Kružkov work [13, see also [11, Chapter VI]. The stability estimates are taken from [7, 14].

First, we study the following Cauchy problem for a scalar non-linear conservation law:

$$
\left\{\begin{array}{l}
\partial_{t} \rho+\operatorname{div}(q(\rho) V(t, x))=0 \\
\rho(0)=\rho_{o} .
\end{array}\right.
$$


Lemma 4.1. Assume that

$$
\begin{aligned}
q & \in \mathbf{C}^{2}\left(\mathbb{R}^{+} ; \mathbb{R}^{+}\right) \text {satisfies } q(0)=0, \\
V & \in \mathbf{C}^{0}\left(\mathbb{R}^{+} \times \mathbb{R}^{d} ; \mathbb{R}^{d}\right) \text { satisfies }\left\{\begin{array}{l}
\operatorname{div} V(t) \in \mathbf{W}^{1,1}\left(\mathbb{R}^{d} ; \mathbb{R}^{d}\right) \\
V(t) \in \mathbf{W}^{1, \infty}\left(\mathbb{R}^{d} ; \mathbb{R}^{d}\right)
\end{array} \text { for all } t \in \mathbb{R}^{+},\right. \\
\rho_{o} & \in\left(\mathbf{L}^{1} \cap \mathbf{L}^{\infty}\right)\left(\mathbb{R}^{d} ; \mathbb{R}^{+}\right) .
\end{aligned}
$$

Then, there exists a unique weak entropy solution $\rho \in \mathbf{C}^{0}\left(\mathbb{R}^{+}, \mathbf{L}^{1}\left(\mathbb{R}^{d} ; \mathbb{R}^{+}\right)\right)$to 4.2$)$.

If furthermore $\rho_{o} \in \mathbf{B V}\left(\mathbb{R}^{d} ; \mathbb{R}\right)$, then $\rho(t) \in \mathbf{B V}\left(\mathbb{R}^{d} ; \mathbb{R}\right)$ for all $t \in \mathbb{R}^{+}$and, denoting $R_{T}=\|\rho(t)\|_{\mathbf{L}^{\infty}\left([0, T] \times \mathbb{R}^{d}\right)}$,

$$
\mathrm{TV}(\rho(t)) \leq \mathrm{TV}\left(\rho_{o}\right) e^{\kappa_{o} t}+C_{d} e^{\kappa_{o} t}\|q\|_{\mathbf{L}^{\infty}\left(\left[0, R_{T}\right]\right)} \int_{0}^{t} \int_{\mathbb{R}^{d}}\|\nabla \operatorname{div} V(\tau, x)\| \mathrm{d} x \mathrm{~d} \tau,
$$

where $\kappa_{o}=(2 d+1)\left\|q^{\prime}\right\|_{\mathbf{L}^{\infty}\left(\left[0, R_{T}\right]\right)}\|\nabla V\|_{\mathbf{L}^{\infty}\left([0, T] \times \mathbb{R}^{d}\right)}$ and $C_{d}=d W_{d}$, with $W_{d}$ defined in (4.4).

Let now $v_{1}, v_{2}, V_{1}, V_{2}$ and $\rho_{o, 1}, \rho_{o, 2}$ satisfy (4.3). Call $\rho_{1}, \rho_{2}$ the solutions to

$$
\left\{\begin{array} { l } 
{ \partial _ { t } \rho _ { 1 } + \operatorname { d i v } ( \rho _ { 1 } v _ { 1 } ( \rho _ { 1 } ) V _ { 1 } ( t , x ) ) = 0 } \\
{ \rho _ { 1 } ( 0 ) = \rho _ { o , 1 } }
\end{array} \quad \text { and } \quad \left\{\begin{array}{l}
\partial_{t} \rho_{2}+\operatorname{div}\left(\rho_{2} v_{2}\left(\rho_{2}\right) V_{2}(t, x)\right)=0 \\
\rho_{2}(0)=\rho_{o, 2}
\end{array}\right.\right.
$$

Then, renaming $R_{T}=\max \left\{\left\|\rho_{1}\right\|_{\mathbf{L}^{\infty}\left([0, T] \times \mathbb{R}^{d}\right)},\left\|\rho_{2}\right\|_{\mathbf{L}^{\infty}\left([0, T] \times \mathbb{R}^{d}\right)}\right\}$,

$$
\begin{aligned}
\left\|\rho_{1}(t)-\rho_{2}(t)\right\|_{\mathbf{L}^{1}} \leq & \left\|\rho_{o, 1}-\rho_{o, 2}\right\|_{\mathbf{L}^{1}} \\
& +t e^{\kappa_{o} t}\left(\left\|q_{2}^{\prime}\right\|_{\mathbf{L}^{\infty}\left(\left[0, R_{T}\right]\right)}\left\|V_{1}-V_{2}\right\|_{\mathbf{L}^{\infty}}+\left\|q_{1}^{\prime}-q_{2}^{\prime}\right\|_{\mathbf{L}^{\infty}\left(\left[0, R_{T}\right]\right)}\left\|V_{1}\right\|_{\mathbf{L}^{\infty}}\right) \\
& \times\left[\operatorname{TV}\left(\rho_{o, 1}\right)+C_{d}\left\|q_{1}\right\|_{\mathbf{L}^{\infty}\left(\left[0, R_{T}\right]\right)} \int_{0}^{t} \int_{\mathbb{R}^{d}}\left\|\nabla \operatorname{div} V_{1}(\tau, x)\right\| \mathrm{d} x \mathrm{~d} \tau\right] \\
& +\left\|q_{1}\right\|_{\mathbf{L}^{\infty}\left(\left[0, R_{T}\right]\right)} \int_{0}^{t} \int_{\mathbb{R}^{d}}\left|\operatorname{div}\left(V_{1}(\tau, x)-V_{2}(\tau, x)\right)\right| \mathrm{d} x \mathrm{~d} \tau \\
& +\left\|q_{1}-q_{2}\right\|_{\mathbf{L}^{\infty}\left(\left[0, R_{T}\right]\right)} \int_{0}^{t} \int_{\mathbb{R}^{d}}\left|\operatorname{div} V_{2}(\tau, x)\right| \mathrm{d} x \mathrm{~d} \tau .
\end{aligned}
$$

Proof. We consider the following steps separately.

Existence. Let $f(t, x, \rho)=q(\rho) V(t, x)$. Then, we have $f \in \mathbf{C}^{0}\left(\mathbb{R}^{+} \times \mathbb{R}^{d} \times \mathbb{R} ; \mathbb{R}^{d}\right)$ and $f(t, \cdot, \cdot) \in \mathbf{C}^{2}\left(\mathbb{R}^{d} \times \mathbb{R} ; \mathbb{R}^{d}\right)$ for any $t \in \mathbb{R}^{+}$. Through elementary computations the following implications can be checked:

$$
\begin{aligned}
& \partial_{\rho} f(t, x, \rho)=q^{\prime}(\rho) V(t, x) \quad \Rightarrow \quad \partial_{\rho} f \quad \text { is } \quad \text { bounded on }[0, T] \times \mathbb{R}^{d} \times[-A, A] \\
& \operatorname{div} f(t, x, \rho)=q(\rho) \operatorname{div} V(t, x) \Rightarrow \operatorname{div} f \in \mathbf{L}^{\infty}\left([0, T] \times \mathbb{R}^{d} \times[-A, A], \mathbb{R}\right) \\
& \partial_{\rho} \operatorname{div} f(t, x, \rho)=q^{\prime}(\rho) \operatorname{div} V(t, x) \Rightarrow \partial_{\rho} \operatorname{div} f \in \mathbf{L}^{\infty}\left([0, T] \times \mathbb{R}^{d} \times[-A, A]\right)
\end{aligned}
$$

for all $A \in \mathbb{R}^{+}$. Hence, [13, Theorem 4] can be applied and the existence of solutions follows.

Maximum principle. Since $q(0)=0, \rho \equiv 0$ is solution to (4.2). The maximum principle of Kružkov [13, Theorem 3] then ensures that $\rho_{o} \geq 0$ implies $\rho(t) \geq 0$ for all $t \geq 0$. 
BV bound. The BV bound follows from [14, Theorem 2.2], which can be applied since $\nabla \partial_{\rho} f=q^{\prime}(\rho) \nabla V(t, x)$, so that $\nabla \partial_{\rho} f \in \mathbf{L}^{\infty}\left([0, T] \times \mathbb{R}^{d} \times[-A, A]\right)$. Moreover, for any $A \geq 0$, $\int_{0}^{T} \int_{\mathbb{R}^{d}}\|\nabla \operatorname{div} f(t, x, \cdot)\|_{\mathbf{L}^{\infty}([-A, A])} \mathrm{d} x \mathrm{~d} t=\|q(\rho)\|_{\mathbf{L}^{\infty}([-A, A])} \int_{0}^{T} \int_{\mathbb{R}^{d}}\|\nabla \operatorname{div} \vec{v}(t, x)\| \mathrm{d} x \mathrm{~d} t<+\infty$. Let $\kappa_{o}=(2 d+1)\left\|q^{\prime}\right\|_{\mathbf{L}^{\infty}\left(\left[0, R_{T}\right]\right)}\|\nabla V\|_{\mathbf{L}^{\infty}\left([0, T] \times \mathbb{R}^{d}\right)}$. Then, for any $t \geq 0$, we have:

$$
\operatorname{TV}(\rho(t)) \leq \operatorname{TV}\left(\rho_{o}\right) e^{\kappa_{o} t}+C_{d} e^{\kappa_{o} t}\|q\|_{\mathbf{L}^{\infty}\left(\left[0, R_{T}\right]\right)} \int_{0}^{t} \int_{\mathbb{R}^{d}}\|\nabla \operatorname{div} V(\tau, x)\| \mathrm{d} x \mathrm{~d} \tau,
$$

with $C_{d}=d W_{d}$, and $W_{d}$ as defined in (4.1).

Stability estimate. The stability estimate follows from [14, Theorem 2.6]. We have, for any $A \geq 0$,

$$
\begin{aligned}
& \int_{0}^{T} \int_{\mathbb{R}^{d}}\left\|\operatorname{div}\left(f_{1}-f_{2}\right)(t, x, \cdot)\right\|_{\mathbf{L}^{\infty}([-A, A])} \mathrm{d} x \mathrm{~d} t \\
= & \left\|q_{1}\left(\rho_{1}\right)\right\|_{\mathbf{L}^{\infty}([-A, A])} \int_{0}^{T} \int_{\mathbb{R}^{d}}\left|\operatorname{div} V_{1}(\tau, x)-\operatorname{div} V_{2}(\tau, x)\right| \mathrm{d} x \mathrm{~d} t \\
& +\left\|q_{1}-q_{2}\right\|_{\mathbf{L}^{\infty}([-A, A])} \int_{0}^{T} \int_{\mathbb{R}^{d}}\left|\operatorname{div} V_{2}(\tau, x)\right| \mathrm{d} x \mathrm{~d} t<+\infty .
\end{aligned}
$$

Then, with $R_{T}=\max \left\{\left\|\rho_{1}\right\|_{\mathbf{L}^{\infty}\left([0, T] \times \mathbb{R}^{d}\right)},\left\|\rho_{2}\right\|_{\mathbf{L}^{\infty}\left([0, T] \times \mathbb{R}^{d}\right)}\right\}$, we get

$$
\begin{aligned}
\left\|\rho_{1}(t)-\rho_{2}(t)\right\|_{\mathbf{L}^{1}} \leq & \left\|\rho_{o, 1}-\rho_{o, 2}\right\|_{\mathbf{L}^{1}} \\
& +t e^{\kappa_{o} t}\left(\left\|q_{2}^{\prime}\right\|_{\mathbf{L}^{\infty}\left(\left[0, R_{T}\right]\right)}\left\|V_{1}-V_{2}\right\|_{\mathbf{L}^{\infty}}+\left\|q_{1}^{\prime}-q_{2}^{\prime}\right\|_{\mathbf{L}^{\infty}\left(\left[0, R_{T}\right]\right)}\left\|V_{1}\right\|_{\mathbf{L}^{\infty}}\right) \\
& \times\left[\operatorname{TV}\left(\rho_{o, 1}\right)+C_{d}\left\|q_{1}\right\|_{\mathbf{L}^{\infty}\left(\left[0, R_{T}\right]\right)} \int_{0}^{t} \int_{\mathbb{R}^{d}}\left\|\nabla \operatorname{div} V_{1}(\tau, x)\right\| \mathrm{d} x \mathrm{~d} \tau\right] \\
& +\left\|q_{2}\right\|_{\mathbf{L}^{\infty}\left(\left[0, R_{T}\right]\right)} \int_{0}^{t} \int_{\mathbb{R}^{d}}\left|\operatorname{div}\left(V_{1}(\tau, x)-V_{2}(\tau, x)\right)\right| \mathrm{d} x \mathrm{~d} \tau \\
& +\left\|q_{1}-q_{2}\right\|_{\mathbf{L}^{\infty}\left(\left[0, R_{T}\right]\right)} \int_{0}^{t} \int_{\mathbb{R}^{d}}\left|\operatorname{div} V_{1}(\tau, x)\right| \mathrm{d} x \mathrm{~d} \tau,
\end{aligned}
$$

completing the proof.

Corollary 4.2. In the same setting as Lemma 4.1, if $q(\rho)=q_{1}(\rho)=q_{2}(\rho)=\rho$ then $q^{\prime}(\rho)=1$, $q^{\prime \prime}(\rho)=0$ and the stability estimate reduces to

$$
\begin{aligned}
\left\|\rho_{1}(t)-\rho_{2}(t)\right\|_{\mathbf{L}^{1}} \leq & \left\|\rho_{o, 1}-\rho_{o, 2}\right\|_{\mathbf{L}^{1}} \\
& +t e^{\kappa_{o} t}\left\|V_{1}-V_{2}\right\|_{\mathbf{L}^{\infty}} \\
& \times\left[\operatorname{TV}\left(\rho_{o, 1}\right)+C_{d}\left\|\rho_{1}\right\|_{\mathbf{L}^{\infty}\left([0, T] \times \mathbb{R}^{d}\right)} \int_{0}^{t} \int_{\mathbb{R}^{d}}\left\|\nabla \operatorname{div} V_{1}(\tau, x)\right\| \mathrm{d} x \mathrm{~d} \tau\right] \\
& +\left\|\rho_{2}\right\|_{\mathbf{L}^{\infty}\left([0, T] \times \mathbb{R}^{d}\right)} \int_{0}^{t} \int_{\mathbb{R}^{d}}\left|\operatorname{div}\left(V_{1}(\tau, x)-V_{2}(\tau, x)\right)\right| \mathrm{d} x \mathrm{~d} \tau .
\end{aligned}
$$




\subsection{Proofs related to Section 2}

Proof of Theorem 2.2, 1. Let $\rho_{o} \in\left(\mathbf{L}^{1} \cap \mathbf{L}^{\infty} \cap \mathbf{B V}\right)\left(\mathbb{R}^{d} ; \mathbb{R}^{n}\right)$ and let $N_{1}=\left\|\rho_{o}\right\|_{\mathbf{L}^{1}}$. Let us introduce a given time $T>0$ and the sphere

$$
\mathcal{X}_{N_{1}}=\left\{\rho \in \mathbf{C}^{0}\left([0, T] ; \mathbf{L}^{1}\left(\mathbb{R}^{d} ; \mathbb{R}^{n}\right)\right): \text { for all } t \in[0, T],\|\rho(t)\|_{\mathbf{L}^{1}} \leq N_{1}\right\},
$$

equipped with the distance induced by the norm $\|\cdot\|_{\mathbf{L}^{\infty}\left([0, T] ; \mathbf{L}^{1}\left(\mathbb{R}^{d} ; \mathbb{R}^{n}\right)\right)}$. Consider first a fixed $i \in\{1, \ldots, n\}$. Choose any $r_{1}, r_{2} \in \mathcal{X}_{N_{1}}$ and denote $\rho_{1}^{i}, \rho_{2}^{i} \in \mathbf{L}^{\infty}\left([0, T] ; \mathbf{L}^{1}\left(\mathbb{R}^{d} ; \mathbb{R}\right)\right)$ the solutions of the Cauchy problems (4.2) with respectively, for $k \in\{1,2\}$,

$$
q_{k}(\rho)=\rho, \quad V_{k}(t, x)=v^{i}\left(r_{k}^{1} * \eta^{1}+\ldots+r_{k}^{n} * \eta^{n}\right) .
$$

Thanks to the properties of $v^{i} \in \mathbf{C}^{2}$ and $\eta$ the hypotheses on $V_{k}$ in Lemma 4.1 are satisfied. Hence, we get existence and uniqueness of a weak entropy solution, Besides, the $\mathbf{L}^{1}$ bound on the solution $\|\rho(t)\|_{\mathbf{L}^{1}}=\left\|\rho_{o}\right\|_{\mathbf{L}^{1}}$ for any $t \geq 0$ ensures that the solution of (4.2)-(4.6) belongs to $\mathcal{X}_{N_{1}}$.

Noting furthermore that

$$
\begin{aligned}
V^{i} & \in \mathbf{L}^{\infty}\left([0, T] \times \mathbb{R}^{d} ; \mathbb{R}^{d}\right), \\
\nabla V^{i} & \in \mathbf{L}^{\infty}\left([0, T] \times \mathbb{R}^{d} ; \mathbb{R}^{d \times d}\right) \cap \mathbf{L}^{\infty}\left([0, T] ; \mathbf{L}^{1}\left(\mathbb{R}^{d} ; \mathbb{R}^{d \times d}\right)\right), \\
\nabla^{2} V^{i} & \in \mathbf{L}^{\infty}\left([0, T] ; \mathbf{L}^{1}\left(\mathbb{R}^{d} ; \mathbb{R}^{d \times d \times d}\right)\right)
\end{aligned}
$$

we can apply [6, Corollary 5.2 and Lemma 5.3] or more directly [14, Theorem 2.2 and Theorem 2.6].

Remark that $N_{1} \geq \sup _{t \in[0, T]}\|r(t)\|_{\mathbf{L}^{1}}$, so that we have the estimate

$$
\begin{aligned}
\left\|\operatorname{div} V^{i}(t)\right\|_{\mathbf{L}^{\infty}} & \leq\left\|\left(v^{i}\right)^{\prime}\right\|_{\mathbf{L}^{\infty}}\|r(t)\|_{\mathbf{L}^{1}}\|\nabla \eta\|_{\mathbf{L}^{\infty}}\left\|\vec{v}^{i}\right\|_{\mathbf{L}^{\infty}}+\left\|v^{i}\right\|_{\mathbf{L}^{\infty}}\left\|\nabla \vec{v}^{i}\right\|_{\mathbf{L}^{\infty}} \\
& \leq\left\|v^{i}\right\|_{\mathbf{W}^{1, \infty}}\left\|\vec{v}^{i}\right\|_{\mathbf{W}^{1, \infty}}\left(N_{1}\|\nabla \eta\|_{\mathbf{L}^{\infty}}+1\right) .
\end{aligned}
$$

Thus, with

$$
K_{1}=\|v\|_{\mathbf{W}^{1, \infty}}\|\vec{v}\|_{\mathbf{W}^{1, \infty}}\left(N_{1}\|\nabla \eta\|_{\mathbf{L}^{\infty}}+1\right),
$$

we obtain by [6, Corollary 5.2]

$$
\left\|\rho^{i}(t)\right\|_{\mathbf{L}^{\infty}} \leq\left\|\rho_{o}^{i}\right\|_{\mathbf{L}^{\infty}} e^{K_{1} t}
$$

Summing over $i=1, \ldots, n$, we obtain

$$
\|\rho(t)\|_{\mathbf{L}^{\infty}} \leq\left\|\rho_{o}\right\|_{\mathbf{L}^{\infty}} e^{K_{1} t} .
$$

We want now to apply Lemma 4.1] or [14, Theorem 2.2]. Note that, with the notations of [14], we have

$$
\kappa_{o}=(2 d+1)\left\|\nabla V^{i}\right\|_{\mathbf{L}^{\infty}} \leq(2 d+1) K_{1}
$$




$$
\begin{aligned}
\left\|\nabla \operatorname{div} V^{i}\right\|_{\mathbf{L}^{1}} \leq & \left\|\left(v^{i}\right)^{\prime \prime}\right\|_{\mathbf{L}^{\infty}}\left(\sum_{j=1}^{n}\left\|\rho^{j}\right\|_{\mathbf{L}^{1}}\left\|\nabla \eta^{j}\right\|_{\mathbf{L}^{\infty}}\right)^{2}\left\|\vec{v}^{i}\right\|_{\mathbf{L}^{1}} \\
& +\left\|\left(v^{i}\right)^{\prime}\right\|_{\mathbf{L}^{\infty}} \sum_{j}\left\|\rho^{j}\right\|_{\mathbf{L}^{1}}\left\|\nabla^{2} \eta^{j}\right\|_{\mathbf{L}^{\infty}}\left\|\vec{v}^{i}\right\|_{\mathbf{L}^{1}} \\
& +2\left\|\left(v^{i}\right)^{\prime}\right\|_{\mathbf{L}^{\infty}} \sum_{j}\left\|\rho^{j}\right\|_{\mathbf{L}^{1}}\left\|\nabla \eta^{j}\right\|_{\mathbf{L}^{\infty}}\left\|\nabla \vec{v}^{i}\right\|_{\mathbf{L}^{1}}+\left\|v^{i}\right\|_{\mathbf{L}^{\infty}}\left\|\nabla^{2} \vec{v}^{i}\right\|_{\mathbf{L}^{1}} \\
\leq & \left\|v^{i}\right\|_{\mathbf{W}^{2, \infty}}\left\|\vec{v}^{i}\right\|_{\mathbf{W}^{2,1}}\left(N_{1}^{2}\|\nabla \eta\|_{\mathbf{L}^{\infty}}^{2}+2 N_{1}\|\nabla \eta\|_{\mathbf{W}^{1, \infty}}+1\right) .
\end{aligned}
$$

Denoting

$$
K_{2}=\|v\|_{\mathbf{W}^{1, \infty}}\|\vec{v}\|_{\mathbf{W}^{1,1}}\left(N_{1}^{2}\|\nabla \eta\|_{\mathbf{L}^{\infty}}^{2}+2 N_{1}\|\nabla \eta\|_{\mathbf{W}^{1, \infty}}+1\right)
$$

we obtain

$$
\operatorname{TV}\left(\rho_{k}^{i}(t)\right) \leq \operatorname{TV}\left(\rho_{o}^{i}\right) e^{(2 d+1) K_{1} t}+t e^{K_{1} t} d W_{d} K_{2}\left\|\rho_{o}^{i}\right\|_{\mathbf{L}^{\infty}} .
$$

Let us now apply Corollary 4.2 or [14, Theorem 2.6]. We obtain

$$
\left\|\rho_{1}^{i}-\rho_{2}^{i}\right\|_{\mathbf{L}^{1}} \leq K t e^{K t}\left\|r_{1}-r_{2}\right\|_{\mathbf{L}^{\infty}\left([0, T] ; \mathbf{L}^{1}\right)}\left[\operatorname{TV}\left(\rho_{o}^{i}\right)+\left(t d W_{d} K_{2}+2+N_{1}\|\nabla \eta\|_{\mathbf{L}^{\infty}}\right)\left\|\rho_{o}^{i}\right\|_{\mathbf{L}^{\infty}}\right]
$$

where

$$
K=\left\|v^{\prime}\right\|_{\mathbf{W}^{1, \infty}}\|\eta\|_{\mathbf{W}^{1, \infty}}\left(\|\vec{v}\|_{\mathbf{L}^{\infty}}+\|\vec{v}\|_{\mathbf{W}^{1,1}}\right) .
$$

Note that $K_{1}, K_{2}$ and $K$ are constants depending on $\|v\|_{\mathbf{W}^{2, \infty}},\|\vec{v}\|_{\mathbf{L}^{\infty}},\|\vec{v}\|_{\mathbf{W}^{2,1}},\|\eta\|_{\mathbf{W}^{2, \infty}}$, $N_{1}$ and $d$ and not on the initial condition $\rho_{o}$. We do not need here precision on the constant so, with a $C$ large enough, not depending on $\rho_{o}$ and $T$, we have, denoting $F(t)=$ $C t e^{C t}\left(\mathrm{TV}\left(\rho_{o}\right)+C(t+1)\left\|\rho_{o}\right\|_{\mathbf{L}^{\infty}}\right)$ and summing over $i=1, \ldots, n$,

$$
\begin{aligned}
\|\rho(t)\|_{\mathbf{L}^{\infty}} & \leq\left\|\rho_{o}\right\|_{\mathbf{L}^{\infty}} e^{C t} \\
\operatorname{TV}(\rho(t)) & \leq \mathrm{TV}\left(\rho_{o}\right) e^{C t}+C t e^{C t}\left\|\rho_{o}\right\|_{\mathbf{L}^{\infty}}, \\
\left\|\left(\rho_{1}-\rho_{2}\right)(t)\right\|_{\mathbf{L}^{1}} & \leq F(t)\left\|r_{1}-r_{2}\right\|_{\mathbf{L}^{\infty}\left([0, T] ; \mathbf{L}^{1}\right)} .
\end{aligned}
$$

Choose $T$ so that $F(T)=1 / 2$. Then, the map $\mathcal{Q}: \mathcal{X}_{N_{1}} \rightarrow \mathcal{X}_{N_{1}}$, defined by $\mathcal{Q}(r)=\rho$, is a contraction. Banach Fixed Point Theorem ensures local in time existence and uniqueness of weak entropy solutions to (1.1)-(2.1).

In order to get global in time existence, we iterate the previous procedure. Starting from time $T_{n}$, we obtain

$$
\begin{aligned}
\left\|\left(\rho_{1}-\rho_{2}\right)(t)\right\|_{\mathbf{L}^{1}} \leq & \left\|r_{1}-r_{2}\right\|_{\mathbf{L}^{\infty}\left([0, t] ; \mathbf{L}^{1}\right)} C\left(t-T_{n}\right) e^{C\left(t-T_{n}\right)} \\
& \times\left(\operatorname{TV}\left(\rho_{o}\right) e^{C T_{n}}+C T_{n} e^{C T_{n}}\left\|\rho_{o}\right\|_{\mathbf{L}^{\infty}}+C\left(1+t-T_{n}\right) e^{C T_{n}}\left\|\rho_{o}\right\|_{\mathbf{L}^{\infty}}\right) .
\end{aligned}
$$

Iteratively, we choose $T_{n+1}>T_{n}$ such that

$$
\left.C\left(T_{n+1}-T_{n}\right) e^{C T_{n+1}}\left(\operatorname{TV}\left(\rho_{o}\right)+C\left(1+T_{n+1}\right)\right)\left\|\rho_{o}\right\|_{\mathbf{L}^{\infty}}\right)=\frac{1}{2} .
$$

Since the sequence $T_{n}$ grows to $+\infty$, we have global in time existence, proving point 1 . 
2. The positivity of the solution directly follows from the fact that $\rho^{i} \equiv 0$ is a solution and from Kružkov Maximum Principle [13, Theorem 3].

3. To prove these estimates, we apply [6, Corollary 5.2] and [14, Theorem 2.2 and Theorem 2.6] to each equation of the system, which is possible since the coupling is only present in the nonlocal term. Let $i \in\{1, \ldots, n\}$. Denote $K_{1}$ as in (4.7), $N_{1}=\left\|\rho_{o}\right\|_{\mathbf{L}^{1}}=\sum_{j=1}^{n}\left\|\rho_{o}^{j}\right\|_{\mathbf{L}^{1}}$ and $V^{i}=v^{i}\left(\rho^{1} * \eta^{1}+\ldots+\rho^{n} * \eta^{n}\right) \vec{v}^{i}(x)$, we have

$$
\|\rho(t)\|_{\mathbf{L}^{\infty}} \leq\left\|\rho_{o}\right\|_{\mathbf{L}^{\infty}} e^{K_{1} t} .
$$

To prove the estimate on the total variation, apply [14, Theorem 2.2]. Denoting $K_{2}$ as in (4.8), we obtain

$$
\operatorname{TV}\left(\rho^{i}(t)\right) \leq e^{(2 d+1) K_{1} t} \operatorname{TV}\left(\rho_{o}^{i}\right)+t e^{(2 d+1) K_{1} t} d W_{d} K_{2}\left\|\rho_{o}^{i}\right\|_{\mathbf{L}^{\infty}} .
$$

Summing over $i=1, \ldots, n$ we obtain the desired bound on $\operatorname{TV}(\rho(t))=\sum_{i=1}^{n} \operatorname{TV}\left(\rho^{i}(t)\right)$.

4. To prove the $\mathbf{L}^{1}$ stability of the solution with respect to initial conditions and parameters, we apply [14, Theorem 2.10]. Denote by $\rho_{k}^{i}$, for $k=1,2$, the solutions to the Cauchy problems

$$
\left\{\begin{array}{l}
\partial_{t} \rho_{k}^{i}+\operatorname{div}\left(\rho_{k}^{i} V_{k}^{i}(t, x)\right)=0, \quad \text { where } \quad V_{k}^{i}(t, x)=v_{k}^{i}\left(\rho_{k}^{1} * \eta_{k}^{1}+\ldots+\rho_{k}^{n} * \eta_{k}^{n}\right) \vec{v}_{k}^{i}(x) . \\
\rho_{k}^{i}(0, \cdot)=\rho_{o, k}^{i}
\end{array}\right.
$$

Note that $\operatorname{div} V_{k}^{i} \in \mathbf{L}^{\infty}\left([0, T] ; \mathbf{L}^{1}\left(\mathbb{R}^{d} ; \mathbb{R}^{d}\right)\right)$ for $k=1,2$, so that the necessary hypotheses hold. Moreover,

$$
\begin{aligned}
& \left\|\operatorname{div}\left(V_{1}^{i}-V_{2}^{i}\right)(t)\right\|_{\mathbf{L}^{1}} \\
\leq & \left\|\left(v_{1}^{i}\right)^{\prime}-\left(v_{2}^{i}\right)^{\prime}\right\|_{\mathbf{L}^{\infty}}\left\|\vec{v}_{1}^{i}\right\|_{\mathbf{L}^{1}}\left\|\rho_{o, 1}\right\|_{\mathbf{L}^{1}}\left\|\nabla \eta_{1}\right\|_{\mathbf{L}^{\infty}}+\left\|v_{1}^{i}-v_{2}^{i}\right\|_{\mathbf{L}^{\infty}}\left\|\operatorname{div} \vec{v}_{1}^{i}\right\|_{\mathbf{L}^{1}} \\
& +\left(\left\|\left(\rho_{1}-\rho_{2}\right)(t)\right\|_{\mathbf{L}^{1}}\left\|\eta_{1}\right\|_{\mathbf{L}^{\infty}}+\left\|\rho_{o, 2}\right\|_{\mathbf{L}^{1}}\left\|\eta_{1}-\eta_{2}\right\|_{\mathbf{L}^{\infty}}\right) \\
& \times\left[\left\|\vec{v}_{1}^{i}\right\|_{\mathbf{L}^{1}}\left\|\left(v_{1}^{i}\right)^{\prime \prime}\right\|_{\mathbf{L}^{\infty}}\left\|\rho_{o, 1}\right\|_{\mathbf{L}^{1}}\left\|\nabla \eta_{1}\right\|_{\mathbf{L}^{\infty}}+\left\|\operatorname{div} \vec{v}_{1}^{i}\right\|_{\mathbf{L}^{1}}\left\|\left(v_{1}^{i}\right)^{\prime}\right\|_{\mathbf{L}^{\infty}}\right] \\
& +\left(\left\|\left(\rho_{1}-\rho_{2}\right)(t)\right\|_{\mathbf{L}^{1}}\left\|\nabla \eta_{1}\right\|_{\mathbf{L}^{\infty}}+\left\|\rho_{o, 2}\right\|_{\mathbf{L}^{1}}\left\|\nabla \eta_{1}-\nabla \eta_{2}\right\|_{\mathbf{L}^{\infty}}\right)\left\|\vec{v}_{1}^{i}\right\|_{\mathbf{L}^{1}}\left\|_{\left(v_{2}^{i}\right)^{\prime}}\right\|_{\mathbf{L}^{\infty}} \\
& +\left\|\operatorname{div}\left(\vec{v}_{1}^{i}-\vec{v}_{2}^{i}\right)\right\|_{\mathbf{L}^{1}}\left\|v_{2}^{i}\right\|_{\mathbf{L}^{\infty}}+\left\|\vec{v}_{1}^{i}-\vec{v}_{2}^{i}\right\|_{\mathbf{L}^{1}}\left\|\left(v_{2}^{i}\right)^{\prime}\right\|_{\mathbf{L}^{\infty}}\left\|\rho_{o, 2}\right\|_{\mathbf{L}^{1}}\left\|\nabla \eta_{2}\right\|_{\mathbf{L}^{\infty}},
\end{aligned}
$$

and

$$
\begin{aligned}
& \left\|\left(V_{1}^{i}-V_{2}^{i}\right)(t)\right\|_{\mathbf{L}^{\infty}} \\
\leq & \left\|v_{1}^{i}-v_{2}^{i}\right\|_{\mathbf{L}^{\infty}}\left\|\vec{v}_{1}^{i}\right\|_{\mathbf{L}^{\infty}}+\left\|v_{2}^{i}\right\|_{\mathbf{L}^{\infty}}\left\|\vec{v}_{1}^{i}-\vec{v}_{2}^{i}\right\|_{\mathbf{L}^{\infty}} \\
& +\left(\left\|\left(\rho_{1}-\rho_{2}\right)(t)\right\|_{\mathbf{L}^{1}}\left\|\eta_{1}\right\|_{\mathbf{L}^{\infty}}+\left\|\rho_{o, 2}\right\|_{\mathbf{L}^{1}}\left\|\eta_{1}-\eta_{2}\right\|_{\mathbf{L}^{\infty}}\right)\left\|\left(v_{1}^{i}\right)^{\prime}\right\|_{\mathbf{L}^{\infty}}\left\|\vec{v}_{1}^{i}\right\|_{\mathbf{L}^{\infty}} .
\end{aligned}
$$

Let us introduce

$$
\alpha=\left\|v_{1}^{\prime \prime}\right\|_{\mathbf{L}^{\infty}}\left\|\eta_{1}\right\|_{\mathbf{L}^{\infty}}\left\|\rho_{o, 1}\right\|_{\mathbf{L}^{1}}\left\|\nabla \eta_{1}\right\|_{\mathbf{L}^{\infty}}\left\|\vec{v}_{1}\right\|_{\mathbf{L}^{1}}+\left\|v_{2}^{\prime}\right\|_{\mathbf{L}^{\infty}}\left\|\nabla \eta_{1}\right\|_{\mathbf{L}^{\infty}}\left\|\vec{v}_{1}\right\|_{\mathbf{L}^{1}}
$$




$$
\begin{aligned}
& +\left\|v_{1}^{\prime}\right\|_{\mathbf{L}^{\infty}}\left\|\eta_{1}\right\|_{\mathbf{L}^{\infty}}\left\|\operatorname{div} \vec{v}_{1}\right\|_{\mathbf{L}^{1}} \\
\beta= & \left\|v_{1}^{\prime \prime}\right\|_{\mathbf{L}^{\infty}}\left\|\rho_{o, 2}\right\|_{\mathbf{L}^{1}}\left\|\rho_{o, 1}\right\|_{\mathbf{L}^{1}}\left\|\nabla \eta_{1}\right\|_{\mathbf{L}^{\infty}}\left\|\vec{v}_{1}\right\|_{\mathbf{L}^{1}}+\left\|v_{1}^{\prime}\right\|_{\mathbf{L}^{1}}\left\|\rho_{o, 2}\right\|_{\mathbf{L}^{1}}\left\|\operatorname{div} \vec{v}_{1}\right\|_{\mathbf{L}^{1}} \\
& +\left\|v_{2}^{\prime}\right\|_{\mathbf{L}^{\infty}}\left\|\rho_{o, 2}\right\|_{\mathbf{L}^{1}}\left\|\vec{v}_{1}\right\|_{\mathbf{L}^{1}} \\
\gamma= & \left\|\operatorname{div} \vec{v}_{1}\right\|_{\mathbf{L}^{1}}+\left\|\rho_{o, 1}\right\|_{\mathbf{L}^{1}}\left\|\nabla \eta_{1}\right\|_{\mathbf{L}^{\infty}}\left\|\vec{v}_{1}\right\|_{\mathbf{L}^{1}} \\
\delta= & \left\|v_{2}^{\prime}\right\|_{\mathbf{L}^{\infty}}\left\|\rho_{o, 2}\right\|_{\mathbf{L}^{1}}\left\|\nabla \eta_{2}\right\|_{\mathbf{L}^{\infty}}+\left\|v_{2}\right\|_{\mathbf{L}^{\infty}}
\end{aligned}
$$

and

$$
\alpha^{\prime}=\left\|v_{1}^{\prime}\right\|_{\mathbf{L}^{\infty}}\left\|\eta_{1}\right\|_{\mathbf{L}^{\infty}}, \quad \beta^{\prime}=\left\|v_{1}^{\prime}\right\|_{\mathbf{L}^{\infty}}\left\|\rho_{o, 2}\right\|_{\mathbf{L}^{1}}\left\|\vec{v}_{1}\right\|_{\mathbf{L}^{\infty}}, \quad \gamma^{\prime}=\left\|\vec{v}_{1}\right\|_{\mathbf{L}^{\infty}}, \quad \delta^{\prime}=\left\|v_{2}\right\|_{\mathbf{L}^{\infty}},
$$

these coefficients being chosen so that

$$
\begin{aligned}
\left\|\operatorname{div}\left(V_{1}^{i}-V_{2}^{i}\right)(\tau)\right\|_{\mathbf{L}^{1}} \leq & \alpha\left\|\left(\rho_{1}-\rho_{2}\right)(\tau)\right\|_{\mathbf{L}^{1}}+\beta\left\|\eta_{1}-\eta_{2}\right\|_{\mathbf{W}^{1, \infty}}+\gamma\left\|v_{1}-v_{2}\right\|_{\mathbf{W}^{1, \infty}} \\
& +\delta\left\|\vec{v}_{1}-\vec{v}_{2}\right\|_{\mathbf{W}^{1,1}}, \\
\left\|\left(V_{1}^{i}-V_{2}^{i}\right)(\tau)\right\|_{\mathbf{L}^{\infty} \leq} \leq & \alpha^{\prime}\left\|\left(\rho_{1}-\rho_{2}\right)(\tau)\right\|_{\mathbf{L}^{1}}+\beta^{\prime}\left\|\eta_{1}-\eta_{2}\right\|_{\mathbf{L}^{\infty}}+\gamma^{\prime}\left\|v_{1}-v_{2}\right\|_{\mathbf{L}^{\infty}} \\
& +\delta^{\prime}\left\|\vec{v}_{1}-\vec{v}_{2}\right\|_{\mathbf{L}^{\infty}} .
\end{aligned}
$$

Hence, we get

$$
\begin{aligned}
& \left\|\left(\rho_{1}^{i}-\rho_{2}^{i}\right)(t)\right\|_{\mathbf{L}^{1}} \\
\leq & \left\|\rho_{o, 1}^{i}-\rho_{o, 2}^{i}\right\|_{\mathbf{L}^{1}}+e^{(2 d+1) K_{1} t}\left[\operatorname{TV}\left(\rho_{o, 1}\right)+t d W_{d} K_{2}\left\|\rho_{o, 1}\right\|_{\mathbf{L}^{\infty}}\right] \int_{0}^{t}\left\|\left(V_{1}^{i}-V_{2}^{i}\right)(\tau)\right\|_{\mathbf{L}^{\infty}} \mathrm{d} \tau \\
& +\max \left\{\left\|\rho_{1}(t)\right\|_{\mathbf{L}^{\infty}},\left\|\rho_{2}(t)\right\|_{\mathbf{L}^{\infty}}\right\} \int_{0}^{t}\left\|\operatorname{div}\left(V_{1}^{i}-V_{2}^{i}\right)(\tau)\right\|_{\mathbf{L}^{1}} \mathrm{~d} \tau \\
\leq & \left\|\rho_{o, 1}^{i}-\rho_{o, 2}^{i}\right\|_{\mathbf{L}^{1}}+A_{\rho}(t) \int_{0}^{t}\left\|\left(\rho_{1}^{i}-\rho_{2}^{i}\right)(\tau)\right\|_{\mathbf{L}^{1}} \mathrm{~d} \tau+t A_{v}(t)\left\|v_{1}-v_{2}\right\|_{\mathbf{W}^{1, \infty}} \\
& +t A_{\eta}(t)\left\|\eta_{1}-\eta_{2}\right\|_{\mathbf{W}^{1, \infty}}+t A_{\vec{v}}(t)\left(\left\|\vec{v}_{1}-\vec{v}_{2}\right\|_{\mathbf{W}^{1,1}}+\left\|\vec{v}_{1}-\vec{v}_{2}\right\|_{\mathbf{L}^{\infty}}\right),
\end{aligned}
$$

where $A_{\rho}, A_{v}, A_{\eta}, A_{\vec{v}}$ are smooth, positive, and increasing, functions of $t$ depending on $d,\|v\|_{\mathbf{W}^{2, \infty}},\|\vec{v}\|_{\mathbf{W}^{1,1}},\|\vec{v}\|_{\mathbf{L}^{\infty}},\|\eta\|_{\mathbf{W}^{1, \infty}}, \operatorname{TV}\left(\rho_{o, 1}\right), \max \left\{\left\|\rho_{o, 1}\right\|_{\mathbf{L}^{\infty}},\left\|\rho_{o, 2}\right\|_{\mathbf{L}^{\infty}}\right\},\left\|\rho_{o, 1}\right\|_{\mathbf{L}^{1}}$ and $\left\|\rho_{o, 2}\right\|_{\mathbf{L}^{1}}$. More precisely, denoting

$$
\begin{aligned}
f(t) & =e^{(2 d+1) K_{1}}\left(\mathrm{TV}\left(\rho_{o, 1}\right)+t d W_{d} K_{2}\left\|\rho_{o, 1}\right\|_{\mathbf{L}^{\infty}}\right), \\
R & =\max \left(\left\|\rho_{o, 1}\right\|_{\mathbf{L}^{\infty}}\left\|\rho_{o, 2}\right\|_{\mathbf{L}^{\infty}}\right), \\
K & =\max _{k \in\{1,2\}}\left\{\left\|v_{k}\right\|_{\mathbf{W}^{1, \infty}}\left\|\vec{v}_{k}\right\|_{\mathbf{W}^{1, \infty}}\left(\left\|\rho_{o, k}\right\|_{\mathbf{L}^{1}}\left\|\nabla \eta_{k}\right\|_{\mathbf{L}^{\infty}}+1\right)\right\}
\end{aligned}
$$

we have

$$
\begin{array}{ll}
A_{\rho}(t)=\alpha^{\prime} f(t)+\alpha R e^{K t}, & A_{\eta}(t)=\beta^{\prime} f(t)+\beta R e^{K t}, \\
A_{v}(t)=\gamma^{\prime} f(t)+\gamma R e^{K t}, & A_{\vec{v}}(t)=\delta^{\prime} f(t)+\delta R e^{K t} .
\end{array}
$$

We conclude applying the Gronwall Lemma, obtaining

$$
\left\|\left(\rho_{1}-\rho_{2}\right)(t)\right\|_{\mathbf{L}^{1}} \leq\left(\left\|\rho_{o, 1}-\rho_{o, 2}\right\|_{\mathbf{L}^{1}}+t A_{\eta}(t)\left\|\eta_{1}-\eta_{2}\right\|_{\mathbf{W}^{1, \infty}}+t A_{v}(t)\left\|v_{1}-v_{2}\right\|_{\mathbf{W}^{1, \infty}}\right.
$$




$$
\begin{aligned}
& \left.+t A_{\vec{v}}(t)\left(\left\|\vec{v}_{1}-\vec{v}_{2}\right\|_{\mathbf{L}^{\infty}}+\left\|\vec{v}_{1}-\vec{v}_{2}\right\|_{\mathbf{W}^{1,1}}\right)\right) \\
& \quad \times\left(1+t\left(f(t) \alpha^{\prime}+R e^{K t} \alpha\right) e^{t f(t) \alpha^{\prime}+t e^{K t} R \alpha}\right),
\end{aligned}
$$

and the stability estimate at 4 . follows.

5. These properties follow through standard computations from the representation formula given in [6, Lemma 5.1].

6. As above, we deduce this further regularity property and the $\mathbf{W}^{2,1}$ estimate from the representation formula in [6, Lemma 5.1].

7. Let $\rho \in \mathbf{C}^{0}\left(\mathbb{R}^{+} ; \mathbf{L}^{1}\left(\mathbb{R}^{d} ; \mathbb{R}^{n}\right)\right)$ be a solution to the initial problem (1.5)-(2.1) such that for all $t \geq 0, \rho(t) \in \mathbf{W}^{2, \infty} \cap \mathbf{W}^{2,1}\left(\mathbb{R}^{d} ; \mathbb{R}^{n}\right)$. Consider now the linearized equations

$$
\left\{\begin{array}{l}
\partial_{t} \sigma^{1}+\operatorname{div}\left(\left(\rho^{1}\left(v^{1}\right)^{\prime}(\rho \star \eta) \sigma \star \eta+\sigma^{1} v^{1}(\rho \star \eta)\right) \vec{v}^{1}(x)\right)=0, \\
\cdots \\
\partial_{t} \sigma^{n}+\operatorname{div}\left(\left(\rho^{n}\left(v^{n}\right)^{\prime}(\rho \star \eta) \sigma \star \eta+\sigma^{n} v^{n}(\rho \star \eta)\right) \vec{v}^{n}(x)\right)=0,
\end{array}\right.
$$

where $\rho=\left(\rho^{1}, \ldots, \rho^{n}\right), \eta=\left(\eta^{1}, \ldots, \eta^{n}\right), \sigma=\left(\sigma^{1}, \ldots \sigma^{n}\right)$ and $\rho \star \eta=\rho^{1} * \eta^{1}+\ldots+\rho^{n} * \eta^{n}$. To prove the existence and uniqueness of weak entropy solutions to this linearized problem, we use the technique that proved to be effective for the initial value problem: let $M_{1}=\left\|\sigma_{o}\right\|_{\mathbf{L}^{1}}$, let $s_{1}, s_{2} \in \mathcal{X}_{M_{1}}$ are fixed functions; we fix the nonlocal term and study the Cauchy problems

$$
\left\{\begin{array}{l}
\partial_{t} \sigma_{k}^{i}+\operatorname{div}\left(\sigma^{i} v^{i}(\rho \star \eta) \vec{v}^{i}(x)\right)=-\operatorname{div}\left(\rho^{i}\left(v^{i}\right)^{\prime}(\rho \star \eta) s_{k} \star \eta \vec{v}^{i}(x)\right) \text { for }\left\{\begin{array}{l}
k=1,2 \\
\sigma_{o, k}^{i}=\sigma_{o}^{i}
\end{array}=1, \ldots, n\right.
\end{array}\right.
$$

We study the map $\mathcal{T}: \mathcal{X}_{M_{1}} \rightarrow \mathcal{X}_{M_{1}}$ defined by $\mathcal{T}(s)=\sigma$. This application is well defined thanks to Kružkov Theorem [13] and thanks to [6, Lemma 5.1]. Indeed, denoting

$$
\begin{array}{ll}
f(t, x, u)=u v^{i}(\rho \star \eta) \vec{v}^{i}(x), & F(t, x, u)=-\operatorname{div}\left(\rho^{i}\left(v^{i}\right)^{\prime}(\rho \star \eta) s_{1} \star \eta \vec{v}^{i}(x)\right), \\
g(t, x, u)=u v^{i}(\rho \star \eta) \vec{v}^{i}(x), & G(t, x, u)=-\operatorname{div}\left(\rho^{i}\left(v^{i}\right)^{\prime}(\rho \star \eta) s_{2} \star \eta \vec{v}^{i}(x)\right),
\end{array}
$$

we have $\partial_{u} f, \partial_{u} g \in \mathbf{L}^{\infty}\left([0, T] \times \mathbb{R}^{d} \times[-U, U]\right), F-\operatorname{div} f, G-\operatorname{div} g \in \mathbf{L}^{\infty}\left([0, T] \times \mathbb{R}^{d} \times[-U, U]\right)$, $\partial_{u}(F-\operatorname{div} f), \partial_{u}(G-\operatorname{div} g) \in \mathbf{L}^{\infty}\left([0, T] \times \mathbb{R}^{d} \times[-U, U]\right)$. Hence Kružkov hypotheses are satisfied. To apply [14, Theorem 2.6], we have now to check that

$$
\begin{aligned}
\nabla \partial_{u} f \in \mathbf{L}^{\infty}\left([0, T] \times \mathbb{R}^{d} \times[-U, U]\right), & \\
\int_{0}^{T} \int_{\mathbb{R}^{d}}\|\nabla(F-\operatorname{div} f)\|_{\mathbf{L}^{\infty}([-U, U])} \mathrm{d} x \mathrm{~d} t & <+\infty, \\
\int_{0}^{T} \int_{\mathbb{R}^{d}}\|F-G-\operatorname{div}(f-g)\|_{\mathbf{L}^{\infty}([-U, U])} \mathrm{d} x \mathrm{~d} t & <+\infty .
\end{aligned}
$$

We have

$$
\begin{aligned}
&\|\nabla F(t, \cdot, u)\|_{\mathbf{L}^{1}} \leq\|\rho(t)\|_{\mathbf{W}^{2, \infty}}\left\|v^{\prime}\right\|_{\mathbf{W}^{1, \infty}}\left\|s_{1}\right\|_{\mathbf{L}^{1}}\|\eta\|_{\mathbf{W}^{2, \infty}}\|\vec{v}\|_{\mathbf{W}^{2,1}} \\
& \times\left[9+6\|\rho(t)\|_{\mathbf{L}^{1}}\|\nabla \eta\|_{\mathbf{L}^{\infty}}+\|\rho(t)\|_{\mathbf{L}^{1}}^{2}\|\nabla \eta\|_{\mathbf{L}^{\infty}}^{2}\right. \\
&\left.+\|\rho(t)\|_{\mathbf{L}^{1}}\left\|\nabla^{2} \eta\right\|_{\mathbf{L}^{\infty}}\right]
\end{aligned}
$$




$$
\begin{aligned}
\|\nabla \operatorname{div} f(t, \cdot, u)\|_{\mathbf{L}^{1}} \leq & |u| K_{2}, \\
\|(F-G)(t, \cdot, u)\|_{\mathbf{L}^{1}} \leq & \|\rho(t)\|_{\mathbf{W}^{1, \infty}}\left\|v^{\prime}\right\|_{\mathbf{W}^{1, \infty}}\|\eta\|_{\mathbf{W}^{1, \infty}}\|\vec{v}\|_{\mathbf{W}^{1,1}}\left(3+\|\rho(t)\|_{\mathbf{L}^{1}}\|\nabla \eta\|_{\mathbf{L}^{\infty}}\right) \\
& \times\left\|\left(s_{1}-s_{2}\right)(t)\right\|_{\mathbf{L}^{1}}, \\
\|\operatorname{div}(f-g)(t, \cdot, u)\|_{\mathbf{L}^{1}}= & 0, \\
\left\|\partial_{u}(f-g)(t, \cdot, u)\right\|_{\mathbf{L}^{\infty}}= & 0 .
\end{aligned}
$$

where $K_{2}$ is defined as in (4.8). Using the estimate on $\|\rho(t)\|_{\mathbf{W}^{1, \infty}}$ obtained in 4. and denoting

$$
K_{4}=\left\|\rho_{o}\right\|_{\mathbf{W}^{1, \infty}}\left\|v^{\prime}\right\|_{\mathbf{W}^{1, \infty}}\|\eta\|_{\mathbf{W}^{1, \infty}}\|\vec{v}\|_{\mathbf{W}^{1,1}}\left(3+\left\|\rho_{o}\right\|_{\mathbf{L}^{1}}\|\nabla \eta\|_{\mathbf{L}^{\infty}}\right)
$$

we obtain

$$
\begin{aligned}
\left\|\sigma_{1}^{i}(t)-\sigma_{2}^{i}(t)\right\|_{\mathbf{L}^{1}} & \leq \int_{0}^{t}\left\|\operatorname{div}\left(\rho^{i}\left(v^{i}\right)^{\prime}(\rho \star \eta)\left(s_{1}-s_{2}\right) \star \eta \vec{v}^{I}\right)\right\|_{\mathbf{L}^{1}} \mathrm{~d} t \\
& \leq t K_{4}(1+C t) e^{C t}\left\|s_{1}-s_{2}\right\|_{\mathbf{L}^{\infty}\left([0, t], \mathbf{L}^{1}\right)} .
\end{aligned}
$$

For $T$ small enough, we obtain that $\mathcal{T}$ is a contraction, proving the local in time existence and uniqueness of solutions by Banach Fixed Point Theorem. We then extend to $+\infty$ the time of existence by iteration of the process.

Denote now by $\rho$, respectively $\rho_{h}$, the solution to the initial problem (1.5)-(2.1) with initial conditions $\rho_{o}$, respectively $\rho_{o}+h \sigma_{o}$. Moreover, call $\sigma$ the solution to the linearized equation (2.2) with initial condition $\sigma_{o}$ and define $z_{h}=\rho+h \sigma$. If $\sigma$ is smooth enough we can write for $z_{h}$ the equation

$$
\partial_{t} z_{h}^{i}+\operatorname{div}\left(z_{h}^{i}\left(v^{i}(\rho \star \eta)+h\left(v^{i}\right)^{\prime}(\rho \star \eta) \sigma \star \eta\right) \vec{v}^{i}(x)\right)=h^{2} \operatorname{div}\left(\sigma^{i}\left(v^{i}\right)^{\prime}(\rho \star \eta) \sigma \star \eta \vec{v}^{i}(x)\right) .
$$

We want now to estimate $\left\|\left(\rho_{h}-z_{h}\right)(t)\right\|_{\mathbf{L}^{1}} / h$. In order to do that, we use [14, Theorem 2.6]. As it is similar to the estimate in the proof of [6, Theorem 2.10], we omit it.

\subsection{Proofs related to Section 3}

Lemma 4.3. In the same setting as Lemma 4.1, if furthermore for a given $R>0$, we have $q(R)=0$, then

$$
\rho_{o} \in[0, R] \quad \Rightarrow \quad \forall t \geq 0, \quad \rho(t) \in[0, R] .
$$

Proof. Since $q(0)=q(R)=0, \rho \equiv 0$ and $\rho \equiv R$ are solutions to (4.2). The maximum principle of Kružkov [13] then ensures that $0 \leq \bar{\rho} \leq R$ implies $0 \leq \rho(t) \leq R$ for all $t \geq 0$.

Proof of Theorem 3.2. Consider the following steps separately.

Existence. Fix an arbitrary positive time $T$, whose precise value will be chosen later. Let $\left(r^{1}, \ldots, r^{n}\right),\left(s^{1}, \ldots, s^{n}\right) \in \mathbf{C}^{0}\left([0, T] ; \mathbf{L}^{1}\left(\mathbb{R}^{d} ;[0, R]^{n}\right)\right)$. For any $i \in\{1, \ldots, n\}$, we define

$$
V^{i}(t, x)=\vec{v}^{i}(x)+\mathcal{I}^{i}\left(r^{1}(t), \ldots, r^{n}(t)\right)(x), \quad W^{i}(t, x)=\vec{v}^{i}(x)+\mathcal{I}^{i}\left(s^{1}(t), \ldots, s^{n}(t)\right)(x) .
$$


As $v$ satisfies $(\mathbf{v})$ and $\mathcal{I}$ satisfies $(\mathbf{I})$, for any $r, s \in \mathbf{C}^{0}\left([0, T], \mathbf{L}^{1}\left(\mathbb{R}^{d},[0, R]^{n}\right)\right)$, we have for any $t \geq 0, V(t), W(t) \in\left(\mathbf{C}^{2} \cap \mathbf{W}^{1, \infty}\right)\left(\mathbb{R}^{d}, \mathbb{R}^{d}\right)$ and $\operatorname{div} V(t), \operatorname{div} W(t) \in \mathbf{W}^{1,1}\left(\mathbb{R}^{d}, \mathbb{R}\right)$. Then, according to Lemma 4.1, there exists $\rho, \sigma \in \mathbf{C}^{0}\left([0, T] ; \mathbf{L}^{1}\left(\mathbb{R}^{d} ; \mathbb{R}_{+}^{n}\right)\right)$, that are the weak entropy solutions to the systems of decoupled equations

$$
\left\{\begin{array} { l } 
{ \partial _ { t } \rho ^ { 1 } + \operatorname { d i v } ( \rho ^ { 1 } v ^ { 1 } ( \rho ^ { 1 } ) V ^ { 1 } ( t , x ) ) = 0 , } \\
{ \cdots } \\
{ \partial _ { t } \rho ^ { n } + \operatorname { d i v } ( \rho ^ { n } v ^ { n } ( \rho ^ { n } ) V ^ { n } ( t , x ) ) = 0 , }
\end{array} \quad \left\{\begin{array}{l}
\partial_{t} \sigma^{1}+\operatorname{div}\left(\sigma^{1} v^{1}\left(\sigma^{1}\right) W^{1}(t, x)\right)=0 \\
\cdots \\
\partial_{t} \sigma^{n}+\operatorname{div}\left(\sigma^{n} v^{n}\left(\sigma^{n}\right) W^{n}(t, x)\right)=0
\end{array}\right.\right.
$$

with initial condition $\rho(0)=\sigma(0)=\rho_{o} \in\left(\mathbf{L}^{1} \cap \mathbf{L}^{\infty}\right)\left(\mathbb{R}^{d} ;[0, R]^{n}\right)$.

Note furthermore that $q^{i}(R)=0$; thus, thanks to Lemma 4.3, that if $\rho$ is a solution of the above equation, then $\rho_{o} \in[0, R]$ implies for all $t \geq 0, \rho(t) \in[0, R]$. Hence, we have invariance of the interval $[0, R]$.

Consider the map

$$
\mathcal{T}:\left\{\begin{array}{ccc}
\mathbf{C}^{0}\left([0, T] ; \mathbf{L}^{1}\left(\mathbb{R}^{d} ;[0, R]^{n}\right)\right) & \longrightarrow & \mathbf{C}^{0}\left([0, T] ; \mathbf{L}^{1}\left(\mathbb{R}^{d} ;[0, R]^{n}\right)\right) \\
r & \mapsto & \rho
\end{array}\right\}
$$

Use Lemma 4.1 and define $\kappa_{o}=\max _{i}\left\{(2 d+1)\left\|\left(q^{i}\right)^{\prime}\right\|_{\mathbf{L}^{\infty}([0, R])}\left\|\nabla V^{i}\right\|_{\mathbf{L}^{\infty}\left([0, T] \times \mathbb{R}^{d}\right)}\right\}$, we get:

$$
\begin{aligned}
&\left\|\rho^{i}(t)-\sigma^{i}(t)\right\|_{\mathbf{L}^{1}} \leq t e^{\kappa_{o} t}\left\|\left(q^{i}\right)^{\prime}\right\|_{\mathbf{L}^{\infty}([0, R])}\left\|V^{i}-W^{i}\right\|_{\mathbf{L}^{\infty}\left([0, t] \times \mathbb{R}^{d}\right)} \\
& \times {\left[\operatorname{TV}\left(\rho_{o}^{i}\right)+C_{d}\left\|q^{i}\right\|_{\mathbf{L}^{\infty}([0, R])} \int_{0}^{t} \int_{\mathbb{R}^{d}}\left\|\nabla \operatorname{div} V^{i}(\tau, x)\right\| \mathrm{d} x \mathrm{~d} \tau\right] } \\
&+\left\|q^{i}\right\|_{\mathbf{L}^{\infty}([0, R])} \int_{0}^{t} \int_{\mathbb{R}^{d}}\left|\operatorname{div}\left(V^{i}(\tau, x)-W^{i}(\tau, x)\right)\right| \mathrm{d} x \mathrm{~d} \tau .
\end{aligned}
$$

Using hypothesis (I), we obtain

$$
\begin{aligned}
& \left\|\rho^{i}(t)-\sigma^{i}(t)\right\|_{\mathbf{L}^{1}} \\
\leq & t C_{I}\|r-s\|_{\mathbf{L}^{\infty}\left([0, t] ; \mathbf{L}^{1}\left(\mathbb{R}^{d} ; \mathbb{R}^{n}\right)\right)} \\
& \times\left[t e^{\kappa_{o} t}\left\|\left(q^{i}\right)^{\prime}\right\|_{\mathbf{L}^{\infty}([0, R])} C_{d}\left\|q^{i}\right\|_{\mathbf{L}^{\infty}([0, R])}\left(\left\|\nabla \operatorname{div} \vec{v}^{i}(x)\right\|_{\mathbf{L}^{1}}+C_{I}\|r\|_{\mathbf{L}^{\infty}\left([0, t], \mathbf{L}^{1}\left(\mathbb{R}^{d} ; \mathbb{R}^{n}\right)\right)}\right)\right. \\
& \left.+e^{\kappa_{o} t}\left\|\left(q^{i}\right)^{\prime}\right\|_{\mathbf{L}^{\infty}([0, R])} \operatorname{TV}\left(\rho_{o}^{i}\right)+\left\|q^{i}\right\|_{\mathbf{L}^{\infty}([0, R])}\right] .
\end{aligned}
$$

Hence, for $T$ small enough, we can apply the Banach Fixed Point Theorem.

Now, using the total variation estimate (4.4), a standard iteration procedure allows to obtain global in time existence.

Total Variation Estimate. To prove the estimate on the total variation, apply Lemma 4.1 and use a procedure entirely similar to that exploited in the proof of Theorem 2.2 . 
Stability. We use [14, Proposition 2.10] to obtain

$$
\begin{aligned}
& \left\|\rho_{1}(t)-\rho_{2}(t)\right\|_{\mathbf{L}^{1}} \\
& \leq\left\|\rho_{o, 1}-\rho_{o, 2}\right\|_{\mathbf{L}^{1}}+e^{\kappa_{o} t}\left(\operatorname{TV}\left(\rho_{o, 1}\right)+t C_{d}\left\|q_{1}\right\|_{\mathbf{L}^{\infty}}\left(C_{I}+\left\|\nabla \operatorname{div} \vec{v}_{1}\right\|_{\mathbf{L}^{1}}\right)\right) \\
& \times\left(\left\|q_{1}^{\prime}\right\|_{\mathbf{L}^{\infty}([0, R])} \int_{0}^{t}\left\|\mathcal{I}\left(\rho_{1}(\tau)\right)-\mathcal{I}\left(\rho_{2}(\tau)\right)\right\|_{\mathbf{L}^{\infty}\left(\mathbb{R}^{d}\right)} \mathrm{d} \tau\right. \\
& \left.+t\left\|q_{1}^{\prime}\right\|_{\mathbf{L}^{\infty}([0, R])}\left\|\vec{v}_{2}-\vec{v}_{1}\right\|_{\mathbf{L}^{\infty}}+t\left(C_{I}+\left\|\vec{v}_{2}\right\|_{\mathbf{L}^{\infty}}\right)\left\|q_{1}^{\prime}-q_{2}^{\prime}\right\|_{\mathbf{L}^{\infty}([0, R])}\right) \\
& +\left\|q_{1}\right\|_{\mathbf{L}^{\infty}([0, R])} \int_{0}^{t}\left\|\operatorname{div} \mathcal{I}\left(\rho_{1}(\tau)\right)-\operatorname{div} \mathcal{I}\left(\rho_{2}(\tau)\right)\right\|_{\mathbf{L}^{1}\left(\mathbb{R}^{d}\right)} \mathrm{d} \tau \\
& +t\left\|q_{1}\right\|_{\mathbf{L}^{\infty}([0, R])}\left\|\operatorname{div} \vec{v}_{2}-\operatorname{div} \vec{v}_{1}\right\|_{\mathbf{L}^{1}}+t\left(C_{I}+\left\|\operatorname{div} \vec{v}_{2}\right\|_{\mathbf{L}^{1}}\right)\left\|q_{1}-q_{2}\right\|_{\mathbf{L}^{\infty}([0, R])} \\
& \leq\left\|\rho_{o, 1}-\rho_{o, 2}\right\|_{\mathbf{L}^{1}} \\
& +t\left[\left(C_{I}+\left\|\vec{v}_{2}\right\|_{\mathbf{L}^{\infty}}\right) e^{\kappa_{o} t}\right. \\
& \times\left(\operatorname{TV}\left(\rho_{o, 1}\right)+t C_{d}\left\|q_{1}\right\|_{\mathbf{L}^{\infty}([0, R])}\left(C_{I}+\left\|\nabla \operatorname{div} \vec{v}_{1}\right\|_{\mathbf{L}^{1}}\right)\right)\left\|q_{1}^{\prime}-q_{2}^{\prime}\right\|_{\mathbf{L}^{\infty}([0, R])} \\
& \left.+\left(C_{I}+\left\|\operatorname{div} \vec{v}_{2}\right\|_{\mathbf{L}^{1}}\right)\left\|q_{1}-q_{2}\right\|_{\mathbf{L}^{\infty}([0, R])}\right] \\
& +t\left[e^{\kappa_{o} t}\left\|q_{1}^{\prime}\right\|_{\mathbf{L}^{\infty}([0, R])}\left(\operatorname{TV}\left(\rho_{o, 1}\right)+t C_{d}\left\|q_{1}\right\|_{\mathbf{L}^{\infty}([0, R])}\left(C_{I}+\left\|\nabla \operatorname{div} \vec{v}_{1}\right\|_{\mathbf{L}^{1}}\right)\right)\left\|\vec{v}_{2}-\vec{v}_{1}\right\|_{\mathbf{L}^{\infty}}\right. \\
& \left.+\left\|q_{1}\right\|_{\mathbf{L}^{\infty}([0, R])}\left\|\operatorname{div}\left(\vec{v}_{2}-\vec{v}_{1}\right)\right\|_{\mathbf{L}^{1}}\right] \\
& +C_{I}\left[e^{\kappa_{o} t}\left\|q_{1}^{\prime}\right\|_{\mathbf{L}^{\infty}([0, R])}\left(\operatorname{TV}\left(\rho_{o, 1}\right)+t C_{d}\left\|q_{1}\right\|_{\mathbf{L}^{\infty}([0, R])}\left(C_{I}+\left\|\nabla \operatorname{div} \vec{v}_{1}\right\|_{\mathbf{L}^{1}}\right)\right)\right. \\
& \left.+\left\|q_{1}\right\|_{\mathbf{L}^{\infty}([0, R])}\right] \\
& \times \int_{0}^{t}\left\|\rho_{1}(\tau)-\rho_{2}(\tau)\right\|_{\mathbf{L}^{1}} \mathrm{~d} \tau
\end{aligned}
$$

Let us denote

$$
\begin{aligned}
a(t)= & t\left[\left(C_{I}+\left\|\vec{v}_{2}\right\|_{\mathbf{L}^{\infty}}\right) e^{\kappa_{o} t}\left(\operatorname{TV}\left(\rho_{o, 1}\right)+t C_{d}\left\|q_{1}\right\|_{\mathbf{L}^{\infty}([0, R])}\left(C_{I}+\left\|\nabla \operatorname{div} \vec{v}_{1}\right\|_{\mathbf{L}^{1}}\right)\right)\right. \\
& \times\left\|q_{1}^{\prime}-q_{2}^{\prime}\right\|_{\mathbf{L}^{\infty}([0, R])} \\
& +\left(C_{I}+\left\|\operatorname{div} \vec{v}_{2}\right\|_{\mathbf{L}^{1}}\right)\left\|q_{1}-q_{2}\right\|_{\mathbf{L}^{\infty}([0, R])}+\left\|q_{1}\right\|_{\mathbf{L}^{\infty}([0, R])}\left\|\operatorname{div}\left(\vec{v}_{2}-\vec{v}_{1}\right)\right\|_{\mathbf{L}^{1}} \\
+ & e^{\kappa_{o} t}\left\|q_{1}^{\prime}\right\|_{\mathbf{L}^{\infty}([0, R])}\left(\operatorname{TV}\left(\rho_{o, 1}\right)+t C_{d}\left\|q_{1}\right\|_{\mathbf{L}^{\infty}([0, R])}\left(C_{I}+\left\|\nabla \operatorname{div} \vec{v}_{1}\right\|_{\mathbf{L}^{1}}\right)\right) \\
\quad & \left.\quad\left\|\vec{v}_{2}-\vec{v}_{1}\right\|_{\mathbf{L}^{\infty}}\right] \\
b(t)= & C_{I}\left[e^{\kappa_{o} t}\left\|q_{1}^{\prime}\right\|_{\mathbf{L}^{\infty}([0, R])}\left(\operatorname{TV}\left(\rho_{o, 1}\right)+t C_{d}\left\|q_{1}\right\|_{\mathbf{L}^{\infty}([0, R])}\left(C_{I}+\left\|\nabla \operatorname{div} \vec{v}_{1}\right\|_{\mathbf{L}^{1}}\right)\right)\right. \\
& \left.+\left\|q_{1}\right\|_{\mathbf{L}^{\infty}([0, R])}\right] .
\end{aligned}
$$

so that, by integration, we get

$$
\left\|\rho_{1}(t)-\rho_{2}(t)\right\|_{\mathbf{L}^{1}} \leq\left(1+t e^{t b(t)}\right)\left(\left\|\rho_{o, 1}-\rho_{o, 2}\right\|_{\mathbf{L}^{1}}+a(t)\right),
$$


which completes the proof.

Acknowledgment: The second author was partially supported by the GNAMPA 2011 project Non Standard Applications of Conservation Laws.

\section{References}

[1] C. Appert-Rolland, P. Degond, and S. Motsch. Two-way multi-lane traffic model for pedestrians in corridors. Networks and Heterogeneous Media, 6(3):351-381, 2011.

[2] N. Bellomo and C. Dogbé. On the modelling of traffic and crowds - a survey of models, speculations, and perspectives. SIAM Review, 2011. To appear.

[3] A. Bressan and G. Guerra. Shift-differentiability of the flow generated by a conservation law. Discrete Contin. Dynam. Systems, 3(1):35-58, 1997.

[4] R. M. Colombo, G. Facchi, G. Maternini, and M. D. Rosini. On the continuum modeling of crowds. In Proceedings of Hyp2008 - the twelfth International Conference on Hyperbolic Problems held in the University of Maryland, College Park, June 2008. To appear.

[5] R. M. Colombo, M. Garavello, and M. Lécureux-Mercier. A class of non-local models for pedestrian traffic. Preprint, 2010.

[6] R. M. Colombo, M. Herty, and M. Mercier. Control of the continuity equation with a non local flow. ESAIM: COCV, 2010.

[7] R. M. Colombo, M. Mercier, and M. D. Rosini. Stability and total variation estimates on general scalar balance laws. Commun. Math. Sci., 7(1):37-65, 2009.

[8] G. Crippa and M. Lécureux-Mercier. Existence and uniqueness of measure solutions for a system of continuity equations with non-local flow. Preprint, 2011.

[9] E. Cristiani, B. Piccoli, and A. Tosin. Multiscale modeling of granular flows with application to crowd dynamics. Multiscale Modeling \& Simulation, 9(1):155-182, 2011.

[10] W. Daamen and S. Hoogendoorn. Experimental research of pedestrian walking behavior. In Transportation Research Board annual meeting 2003, pages 1-16. National Academy Press, 2007.

[11] C. M. Dafermos. Hyperbolic conservation laws in continuum physics, volume 325 of Grundlehren der Mathematischen Wissenschaften [Fundamental Principles of Mathematical Sciences]. Springer-Verlag, Berlin, second edition, 2005.

[12] D. Helbing, P. Molnár, I. Farkas, and K. Bolay. Self-organizing pedestrian movement. Environment and Planning B: Planning and Design, 28:361-383, 2001.

[13] S. N. Kružkov. First order quasilinear equations with several independent variables. Mat. Sb. (N.S.), 81 (123):228-255, 1970.

[14] M. Lécureux-Mercier. Improved stability estimates on general scalar balance laws. To appear on J. of Hyperbolic Differential Equations, 2011.

[15] R. Levy and M. Shearer. The motion of a thin liquid film driven by surfactant and gravity. SIAM J. Appl. Math., 66(5):1588-1609, 2006. 